\title{
Eine elektronenspektroskopische Deutung der Konformation dimerer Porphinderivate mit Hilfe des Modells der Wechselwirkung ausgedehnter Dipole
}

\author{
Bernd von Maltzan \\ Institut für Organische Chemie der Freien Universität Berlin \\ Z. Naturforsch. 40 a, 389-420 (1985); eingegangen am 21. Januar 1985 \\ An Electron Spectroscopical Interpretation of the Conformation of Dimeric Porphine Derivatives \\ by the Extended Dipole Model
}

Derivatives of meso-tetramethylporphine (TMP; $\mathbf{1} \mathbf{a - h}$ ), which are analogs of diphenylmethane and triphenylmethane, and methylene bridged dimers $(2 \mathrm{a}-\mathbf{3 i})$ are examined by electron spectra. The dimers show an intense splitting of the Soretband, which is the result of the conformation of the exciton coupled porphine dimer. The extended dipole model developed by H. Kuhn, H. D. Försterling et al. for aggregates of dye molecules, is used for the interpretation of this interaction. An excellent agreement between the observed and the predicted splitting of the Soret band is found, based on the energetically favoured conformation. Furthermore, this model is examined for literature known examples of porphine dimers. The splitting of the Soretband is also influenced by the solvent, which is explained by changes of the conformation.

Die Kenntnis der Struktur von Porphin- bzw. Chlorophyllaggregaten ist eine wichtige Voraussetzung für den Einblick in den Mechanismus des Photosyntheseapparates [1-6], vor allen Dingen für den Transport der Anregungsenergie. Eine vielverwendete aufschlußreiche Methode für eine Aussage über die Lage der Porphinringe zueinander ist die Kernresonanz- sowie die Elektronenspinresonanzspektroskopie [9-12]. Untersucht wird aber auch die Möglichkeit, auf Grund der Elektronenspektren eine Aussage über die Form von Porphinaggregaten zu machen $[7,8]$.

Als besonders geeignet erscheinen dafür miteinander kovalent verknüpfte Porphine, die zwangsweise aggregierte Moleküle darstellen. So sind in letzter Zeit eine Reihe dimerer und trimerer Porphine synthetisiert worden, bei denen die Porphinringe durch bewegliche kovalente Brücken sowohl cyclophanartig übereinander [11-18], wie auch nebeneinander zu liegen kamen [19-26]. Ebenso sind einige $\mu$-oxo-verknüpfte Ge-, Sn- [27] und Sc-Metallkomplexe [28], sowie auch Polymere erwähnt, in denen die Porphinringe durch axiale Substituenten am zentralen Metallion sandwichartig zusammengehalten werden [27, 29].

Sonderdruckanforderungen an B. v. Maltzan, Institut für Organische Chemie der Freien Universität Berlin, Takustr. 3, D-1000 Berlin 33.
Für die nebeneinander verknüpften Porphindimere wurde von M. Gouterman und Mitarbeitern [30] und für die cyclophanartigen von Chang eine grobe qualitative Interpretation angedeutet [12]. Eine genauere Deutung der Spektren, die den hier beschriebenen teilweise ähnlich sind, konnte nicht gegeben werden, da hierfür eine detaillierte Kenntnis der Konformation dieser Moleküle nötig ist $[11,30]$. Für sandwichartige $\mu$-oxo-verknüpfte dimere Sc-Komplexe wurde eine Interpretation mit Hilfe des Excitonen-Modells vorgenommen und die Lage der Banden berechnet [28].

Da quantenchemische Rechnungen für die dimeren Porphine auf Grund ihres Umfanges nur schwer durchführbar und auch nachvollziehbar sind, ist es gerechtfertigt, nach einfacheren Modellen zu suchen, auch wenn diese auf semiempirischen halbquantitativen Methoden beruhen.

In dieser Arbeit wird nun eine Möglichkeit angedeutet, die Elektronenspektren dimerer Porphine, die über eine kurze Brücke miteinander verknüpft sind, in Abhängigkeit ihrer Abstände und ihrer Konformation zueinander zu interpretieren. Dafür wird das von Czikkely, Försterling und Kuhn abgeleitete einfache Modell [31] der Wechselwirkung ausgedehnter Dipole aggregierter Farbstoffmoleküle verwendet. Wie mit Hilfe von kovalent verbundenen dimeren Anthracenderivaten, deren Konformation mehr oder weniger exakt bekannt war, ge-

0340-4811 / $85 / 0400-0389 \$ 01.30 / 0$. - Please order a reprint rather than making your own copy. 
zeigt werden konnte, liefert dieses einfach zu handhabende Modell eine gute Übereinstimmung von experimentellen und berechneten Absorptionsbanden [32].

Die Elektronenspektren der neu synthetisierten Porphindimere $\mathbf{3} \mathbf{a}-\mathbf{h}$ [33] ergaben eine auffallende Aufspaltung der Soret-Bande. Dies wurde ebenso an ähnlich verknüpften Porphindimeren beobachtet, wenn auch nicht in dieser ausgeprägten Form [30], da die Soret-Bande lediglich bei der protonierten Form der Dimeren zwei getrennte Einzelbanden zeigt. Die schon früher beobachtete Verbreiterung dieser Bande bei den unsymmetrischen Diporphinylmethanen $\mathbf{2}$ b und $\mathbf{2} \mathbf{c}$ wurde anfänglich als Summe zweier dicht beieinander liegender Einzelbanden interpretiert, wobei die $425 \mathrm{~nm}$-Bande dem rein meso-substituierten und die bei $431 \mathrm{~nm}$ liegende Bande dem zusätzlich in $\beta$-Position substituierten Porphinring zugeordnet wurde [23]. Diese Interpretation trifft so nicht zu, da bei den symmetrischen Diporphinylmethanen der Struktur 3 dann nur eine Bande erscheinen dürfte.

\section{Spektroskopische Beobachtungen}

Wie der Tab. 1 und der Abb. 1 zu entnehmen ist, zeigen die Elektronenspektren der monomeren meso-Tetramethylporphinderivate $\mathbf{1} \mathbf{d}-\mathbf{h}$ sowie der Stammverbindung 1a-c keinen außergewöhnlichen Bandenverlauf. Dieser gleicht sehr stark dem des meso-Tetraphenylporphin bzw. meso-Tetratolylporphins [34]. Dagegen ist die Soret-Bande der dimeren Porphinderivate sowohl der Struktur 2 wie auch der Struktur $\mathbf{3}$ in zwei deutlich voneinander getrennte Teilbanden aufgespalten. Bei den dimeren di-Nickelkomplexen ist diese Aufspaltung nicht so stark ausgeprägt. Dies ist darauf zurückzuführen, daß die Halbwertsbreite der einfachen Soret-Bande des monomeren Nickelkomplexes $\mathbf{1}$ b bereits $19 \mathrm{~nm}$ ist und mit größer werdendem Substituenten an der $\beta$-Position bis auf $22 \mathrm{~nm}$ bei $\mathbf{1} \mathbf{g}$ ansteigt. Die Halbwertsbreite der Soret-Bande der monomeren Neutralbasen $\mathbf{1}$ a, $\mathbf{1} \mathbf{d}$ und $\mathbf{1} \mathbf{f}$ liegt erheblich unter diesem Wert, steigt aber ebenfalls mit größer werdenden Substituenten von 11,5 bis $15 \mathrm{~nm}$ an; in der proto-

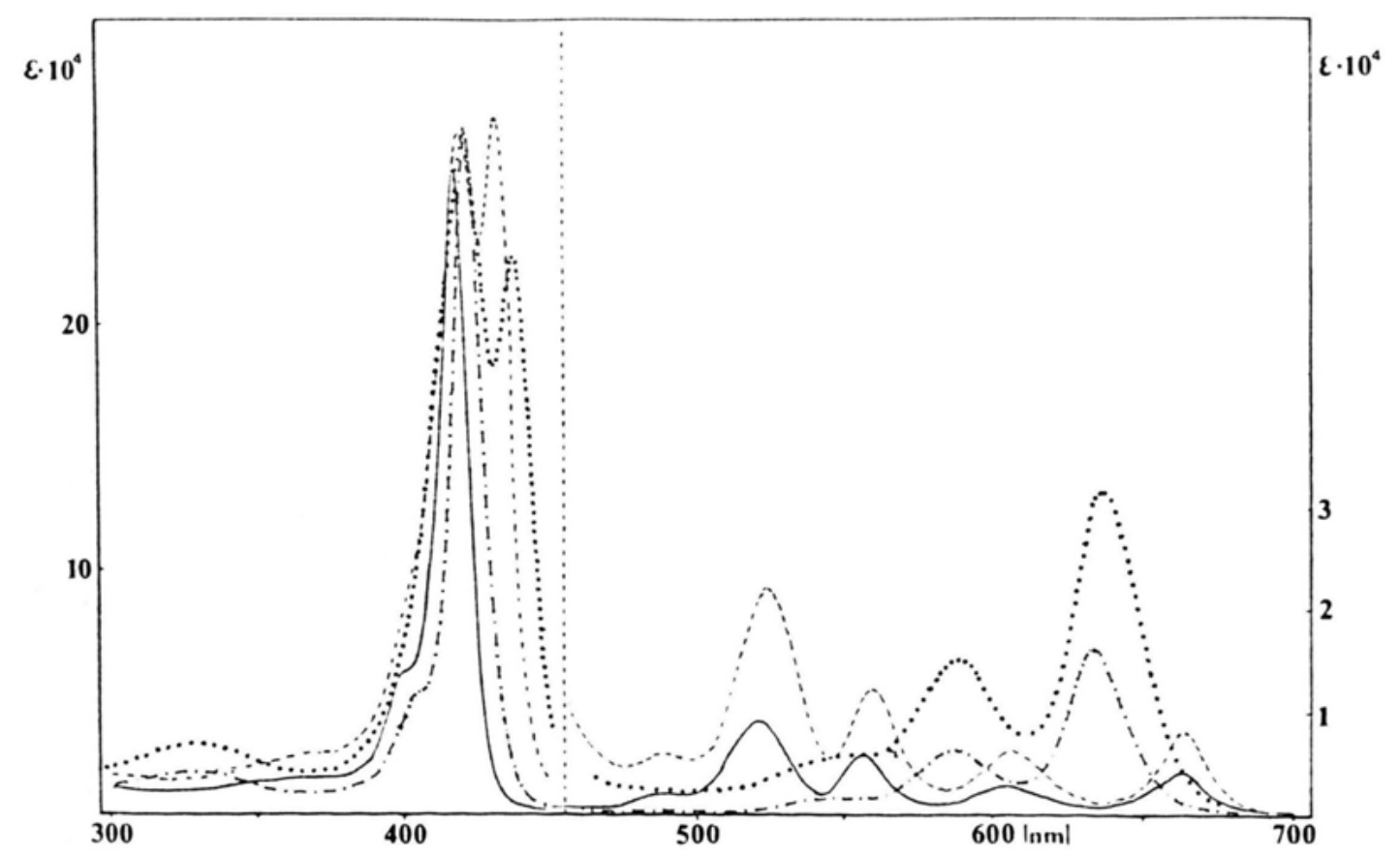

Abb. 1 a. Elektronenspektren des (Phenyl) (porphinyl) methans 1 d $\left(-\frac{-}{-}\right.$ ), seines Kations $(\cdot-\cdot-\cdot)$ des 1,1-Diporphinylethans $3 \mathbf{d}(\ldots)$ und seines Kations (...) in $\mathrm{CHCl}_{3}$ bzw. in $\mathrm{CHCl}_{3} / 2 \%$ Trifluoressigsäure. 


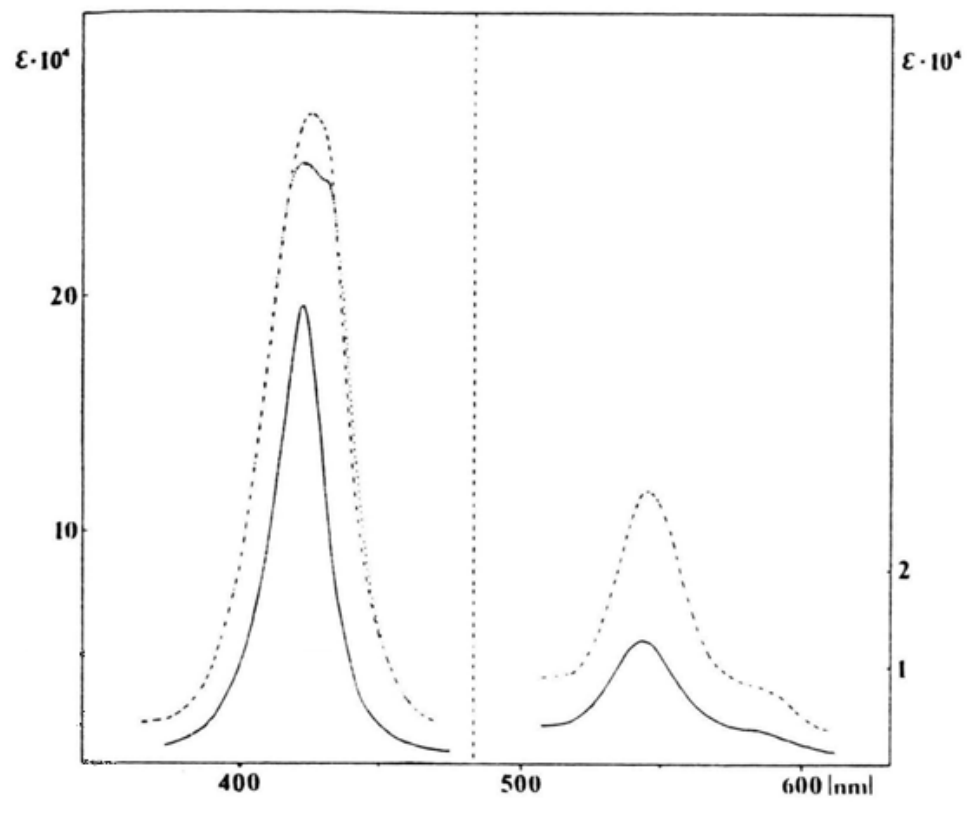

Abb. 1b. Elektronenspektren der Nickelkomplexe des (Diphenyl) (porphinyl)methans $1 \mathrm{~g}(-)$ des symmetrischen Diporphinylmethans $\mathbf{3 b}(-.$.$) und die Soret-$ Bande des (Phenyl)(diporphinyl)-methans $3 \mathrm{~h}(\cdots \cdots)$, (die Bande bei $545 \mathrm{~nm}$ ist gleich der von $\mathbf{3}$ b) in $\mathrm{CHCl}_{3}$.

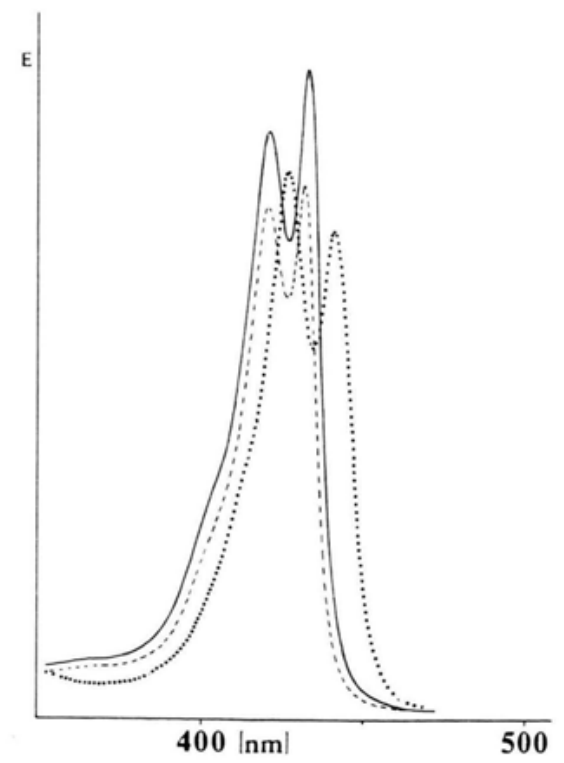

Abb. 1c. Elektronenspektrum im Bereich der Soret-Bande des unsymmetrischen Diporphinylmethans 2 a $(-)$, seines vierwertigen Kations $(\cdots)$ und des symmetrischen Diporphinylmethans $3 \mathbf{a}(---)$. (Die Konzentration von $\mathbf{3} \mathbf{a}$ ist etwas geringer als die von $\mathbf{2}$ a, um die enorme Ähnlichkeit deutlich zu zeigen; Lsgm.: $\mathrm{CHCl}_{3}$ bzw. $\mathrm{CHCl}_{3} / 1 \%$ $\mathrm{CF}_{3} \mathrm{COOH}$ ). nierten Form sind diese nur geringfügig um $1 \mathrm{~nm}$ größer.

Somit ist erklärlich, warum die Aufspaltung der Soret-Bande bei den mono-metallierten und metallfreien Dimeren 2a, 3a, 3c, 3d, 3f und 3 $\mathrm{g}$ ausgeprägter ist. Wesentlicher hierfür ist, daß die Differenz der Teilbanden in den dimeren Neutralbasen auf $\Delta \lambda \sim 12 \mathrm{~nm}$ und in deren protonierten Form auf $\Delta i \sim 16 \mathrm{~nm}$ zunimmt.

Beeinflußt wird diese Differenz der beiden Teilbanden u. a. vom Substituenten an der Methylenbrücke von $\mathbf{3} \mathbf{a}-\mathbf{h}$ : mit größerem Rest $R$ nimmt sie zu. Es verändert sich aber auch die Form der Bande. Da diese aus der Überlagerung mindestens zweier Kurven resultiert und schon bei geringer gegenseitiger Verschiebung der Einzelbanden eine qualitative Änderung in der Gesamtform erfährt (wie später gezeigt), stellt sie ein empfindliches $\mathrm{Ma} ß$ für strukturelle Einflüsse dar. Dies zeigt sich sowohl bei den di-Ni-Komplexen $\mathbf{3}$ b, $\mathbf{3}$ e und $\mathbf{3}$ h (s. Abb. 1 b), den monokomplexierten Dimeren $3 \mathbf{c}$ und $3 \mathbf{f}$ (Abb. 2), wie auch bei den Neutralbasen $\mathbf{3 a}$ a, $\mathbf{3 d}$ und $\mathbf{3} \mathbf{g}$. So ist die Soret-Bande von $\mathbf{3} \mathbf{b}$ fast symmetrisch, zeigt nur andeutungsweise eine Aufspaltung, aber die Halbwertsbreite beträgt $32 \mathrm{~nm}$. Sie läßt sich aus zwei Einzelbanden (bei $421 \mathrm{~nm}$ und $428 \mathrm{~nm}$ ) mit der Halbwertsbreite von $\lambda_{1 / 2}=25 \mathrm{~nm}$ zusammensetzen. 


\begin{tabular}{|c|c|c|c|c|c|c|c|c|c|c|c|}
\hline \multirow{3}{*}{$\begin{array}{l}\begin{array}{l}\text { Verbin- } \\
\text { dung }\end{array} \\
\mathbf{1 a}\end{array}$} & Solvens & \multicolumn{3}{|c|}{$\lambda_{\max } \mathrm{nm}(\lg \varepsilon)$} & \multicolumn{7}{|l|}{ Soret } \\
\hline & $\begin{array}{l}\mathrm{CHCl}_{3} \\
\mathrm{CHCl}_{3} /\end{array}$ & $\begin{array}{l}301 \\
-\end{array}$ & $\begin{array}{l}363,5(4,18) \\
320(4,62)\end{array}$ & $\begin{array}{l}400(4,78) \\
404(4,86)\end{array}$ & $\begin{array}{l}416,5(5,42) \\
421(5,54)\end{array}$ & {$[11,5]^{b}$} & $\begin{array}{l}484(3,51) \\
-\end{array}$ & $\begin{array}{l}519,5(4,03) \\
-\end{array}$ & $\begin{array}{l}556(3,88) \\
584(3,98)\end{array}$ & $\begin{array}{l}602(3,55) \\
633(4,34)\end{array}$ & $\begin{array}{l}663(3,70) \\
-\end{array}$ \\
\hline & $\begin{array}{l}\mathrm{CF}_{3} \mathrm{CO}_{2} \mathrm{H} \\
\mathrm{H}_{2} \mathrm{SO}_{4} \\
\mathrm{HCl} \text { (konz.) } \\
\mathrm{CHCl}_{3} / \mathrm{EtO} \\
+0,5 \% \mathrm{HCl}\end{array}$ & ${ }_{\mathrm{H}}^{-}$ & $324(4,20)$ & $399(4,74)$ & $\begin{array}{l}414(5,56) \\
421 \\
428,7\end{array}$ & $\begin{array}{l}{[11]} \\
{[12,8]} \\
{[12,8]}\end{array}$ & $\begin{array}{l}- \\
\overline{-}\end{array}$ & $\begin{array}{l}540(3,80) \\
552\end{array}$ & $\begin{array}{l}578(3,80) \\
585,5 \\
587\end{array}$ & $\begin{array}{l}627(4,25) \\
634 \\
638,5\end{array}$ & - \\
\hline \multicolumn{3}{|c|}{$\mathrm{TMPH}_{4} \mathrm{Cl}_{2} \mathrm{CHCl}_{3}$} & 348 & 417 & 431 & {$[11]$} & - & 554 & 594 & 642 & - \\
\hline $1 \mathrm{~b}$ & $\begin{array}{l}\mathrm{CHCl}_{3} \\
\mathrm{CH}_{3} \mathrm{CN}\end{array}$ & $\begin{array}{l}300(4,06) \\
199(4,75)\end{array}$ & $\begin{array}{c}332(3,97) \\
235,5(4,2) \quad 250(\end{array}$ & $\begin{array}{l}- \\
(3,93)\end{array} 295(4$. & $\begin{array}{l}\text { 418 }(5,27) \\
01) 326(3,95)\end{array}$ & $\begin{array}{l}{[19]} \\
412(5,245)\end{array}$ & $\begin{array}{l}- \\
-\end{array}$ & $\begin{array}{l}539(4,09) \\
534(4,03)\end{array}$ & $\overline{\text { Schulter }}$ & $\overline{-}$ & - \\
\hline $1 \mathrm{c}$ & $\mathrm{CHCl}_{3}$ & $-{ }^{a}$ & - & - & $418(5,28)$ & {$[16]$} & $494(3,30)$ & $533(4,09)$ & $566(3,48)$ & - & - \\
\hline \multirow[t]{2}{*}{$1 \mathrm{~d}$} & $\begin{array}{l}\mathrm{CHCl}_{3} \\
\mathrm{CHCl}_{3} / \\
\mathrm{CF}_{3} \mathrm{CO}_{2} \mathrm{H}\end{array}$ & $302(3,96)$ & $\begin{array}{l}363(4,14) \\
327,5(4,21)\end{array}$ & $\begin{array}{l}400(4,76) \\
404(4,69)\end{array}$ & $\begin{array}{l}417,5(5,42) \\
421(5,45)\end{array}$ & $\begin{array}{l}{[11,5]} \\
{[12,5]}\end{array}$ & $\begin{array}{l}485(3.31) \\
-\end{array}$ & $\begin{array}{l}520(3,96) \\
535(3,10)\end{array}$ & $\begin{array}{l}556(3,77) \\
586(3,80)\end{array}$ & $\begin{array}{l}603(3,42) \\
633(4.21)\end{array}$ & $\begin{array}{l}663(3,60) \\
-\end{array}$ \\
\hline & $\mathrm{H}_{2} \mathrm{SO}_{4}$ & - & $331(4,29)$ & $400(4,73)$ & $418(5,38)$ & {$[13,5]$} & - & - & $580(3,68)$ & $630(4,14)$ & - \\
\hline $1 \mathrm{e}$ & $\mathrm{CHCl}_{3}$ & $302(4,10)$ & $333(4,03)$ & - & $421(5,27)$ & {$[21,2]$} & - & $543,5(4,13)$ & - & - & - \\
\hline \multirow[t]{2}{*}{$1 \mathrm{f}$} & $\begin{array}{l}\mathrm{CHCl}_{3} \\
\mathrm{CHCl}_{3} / \\
\mathrm{CF}_{3} \mathrm{CO}_{2} \mathrm{H}\end{array}$ & $\begin{array}{l}305(4,09) \\
-\end{array}$ & $\begin{array}{l}365(4,18) \\
327(4,25)\end{array}$ & $\begin{array}{l}402(4,78) \\
409(4,75)\end{array}$ & $\begin{array}{l}420(5,43) \\
427(5,45)\end{array}$ & $\begin{array}{l}{[13]} \\
{[15]}\end{array}$ & $\begin{array}{l}488(3,45) \\
-\end{array}$ & $\begin{array}{l}522,5(4,03) \\
545(3,47)\end{array}$ & $\begin{array}{l}558(3,79) \\
589(3,93)\end{array}$ & $\begin{array}{l}605(3,48) \\
638(4,25)\end{array}$ & $\begin{array}{l}663(3,65) \\
-\end{array}$ \\
\hline & $\mathrm{H}_{2} \mathrm{SO}_{4}$ & - & $325(4,29)$ & 402 (Sch) & $420,5(5,41)$ & {$[15,5]$} & - & - & $584(3,64)$ & $633(4,16)$ & - \\
\hline $1 \mathrm{~g}$ & $\mathrm{CHCl}_{3}$ & $301(4,06)$ & $339(4,03)$ & - & $423(5,29)$ & {$[22]$} & - & $544,5(4,10)$ & - & - & - \\
\hline $1 \mathrm{~h}$ & $\begin{array}{l}\mathrm{CHCl}_{3} \\
\mathrm{CH}_{3} \mathrm{CN}\end{array}$ & $\begin{array}{l}300(4,07) \\
199(4,89)\end{array}$ & $\begin{array}{l}344(4,02) \\
235(4,26)\end{array}$ & $295(4$, & $\begin{array}{l}420(5,20) \\
\text { 2) } 325(3,95)\end{array}$ & $\begin{array}{l}{[21,5]} \\
414(5,17)\end{array}$ & - & $\begin{array}{l}541(4,12) \\
537(4,03)\end{array}$ & - & $\overline{-}$ & $\begin{array}{l}- \\
-\end{array}$ \\
\hline 2 a & $\begin{array}{l}\mathrm{CHCl}_{3} \\
\mathrm{CHCl}_{3} / \\
\mathrm{CF}_{3} \mathrm{CO}_{2} \mathrm{H}\end{array}$ & $\begin{array}{l}303(4,22) \\
-\end{array}$ & $\begin{array}{l}370(4,36) \\
330(4,52)\end{array}$ & - & $\begin{array}{l}419(5,35) \\
425(5,44)\end{array}$ & $\begin{array}{l}432(5,40) \\
440(5,39)\end{array}$ & $\begin{array}{l}487(3,72) \\
-\end{array}$ & $\begin{array}{l}522(4,29) \\
-\end{array}$ & $\begin{array}{l}557(4,05) \\
586(4,13)\end{array}$ & $\begin{array}{l}602,5(3,78) \\
636(4,45)\end{array}$ & $662(3,86)$ \\
\hline $2 \mathrm{~b}$ & $\mathrm{CHCl}_{3}$ & $302(4,28)$ & $333(4,22)$ & - & $425(5,29)$ & $431(5,30)$ & - & $542(4,37)$ & - & - & - \\
\hline $2 \mathrm{c}$ & $\mathrm{CHCl}_{3}$ & $-\mathrm{a}$ & - & - & $423(5,29)$ & $431(5.32)$ & $497(3,08)$ & $537(4,38)$ & $568(3,71)$ & - & - \\
\hline \multirow[t]{2}{*}{$3 \mathbf{a}$} & $\begin{array}{l}\mathrm{CHCl}_{3} \\
\mathrm{CHCl}_{3} / \\
\mathrm{CF}_{3} \mathrm{CO}_{2} \mathrm{H}\end{array}$ & $303(4,28)$ & $\begin{array}{l}367,5(4,45) \\
329(4,46)\end{array}$ & - & $\begin{array}{l}419(5,45) \\
423(5,47)\end{array}$ & $\begin{array}{l}430(5,46) \\
437,5(5,40)\end{array}$ & $\begin{array}{l}486(3,74) \\
-\end{array}$ & $\begin{array}{l}522(4,30) \\
-\end{array}$ & $\begin{array}{l}557(4,09) \\
587,5(4,21)\end{array}$ & $\begin{array}{l}605(3,84) \\
637,5(4,51)\end{array}$ & $663(3,88)$ \\
\hline & $\mathrm{H}_{2} \mathrm{SO}_{4}$ & - & $334(4,60)$ & - & $417,5(5,52)$ & $432(5,43)$ & - & - & $584(4,12)$ & $632(4,53)$ & - \\
\hline $3 \mathbf{b}$ & $\mathrm{CHCl}_{3}$ & $302(4,37)$ & $333(4,31)$ & - & $426(5,44)$ & {$[32]$} & - & $544(4,46)$ & - & - & - \\
\hline $3 \mathrm{c}$ & $\begin{array}{l}\mathrm{CHCl}_{3} \\
\mathrm{CHCl}_{3} / \\
\mathrm{CF}_{3} \mathrm{CO}_{2} \mathrm{H}\end{array}$ & $303(4,25)$ & $\overline{330}(4,37)$ & - & $\begin{array}{l}419(5,42) \\
423,5(5,44)\end{array}$ & $\begin{array}{l}429(5,41) \\
432,5(5,45)\end{array}$ & $\begin{array}{l}524(4,17) \\
-\end{array}$ & $\begin{array}{l}540(4,15) \\
542(4,15)\end{array}$ & $\begin{array}{l}555(4,13) \\
587,5(4,03)\end{array}$ & $\begin{array}{l}602,5(3,60) \\
636(4,28)\end{array}$ & ${ }_{-}^{663}(3,60)$ \\
\hline \multirow[t]{2}{*}{3 d } & $\begin{array}{l}\mathrm{CHCl}_{3} \\
\mathrm{CHCl}_{3} / \\
\mathrm{CF}_{3} \mathrm{CO}_{2} \mathrm{H}\end{array}$ & $\begin{array}{l}301(4,27) \\
-\end{array}$ & $\begin{array}{l}367(4,39) \\
327(4,51)\end{array}$ & - & $\begin{array}{l}419,5(5,44) \\
426,5(5,44)\end{array}$ & $\begin{array}{l}432(5,45) \\
441,5(5.35)\end{array}$ & $\begin{array}{l}488(3,79) \\
-\end{array}$ & $\begin{array}{l}523(4,35) \\
546(3,80)\end{array}$ & $\begin{array}{l}559(4,09) \\
588(4,20)\end{array}$ & $\begin{array}{l}605(3,78) \\
637(4,51)\end{array}$ & $664(3,90)$ \\
\hline & $\mathrm{H}_{2} \mathrm{SO}_{4}$ & - & $340(4,68)$ & - & $419(5,44)$ & $433(5,32)$ & - & - & $586(4,08)$ & $634(4,43)$ & - \\
\hline
\end{tabular}




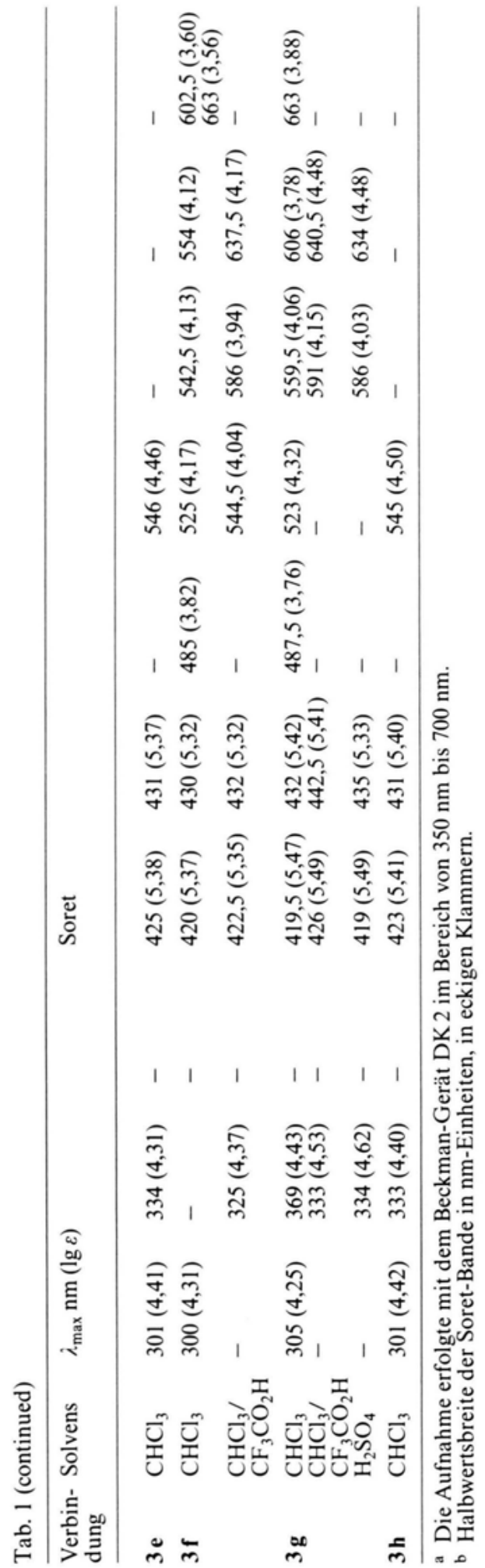

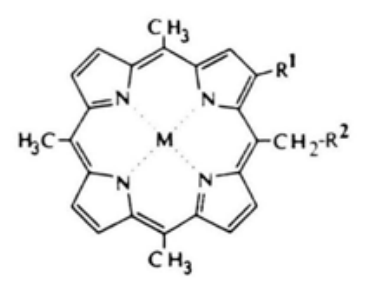

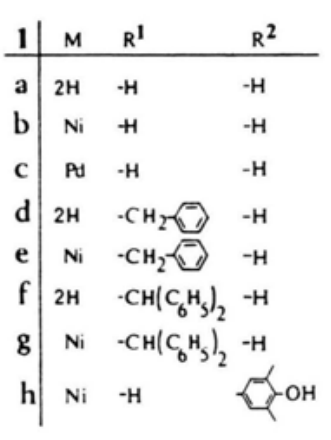

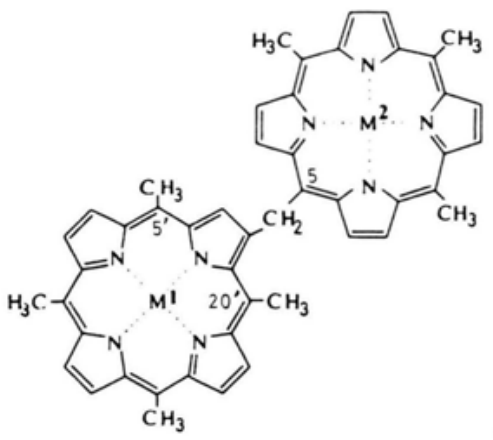

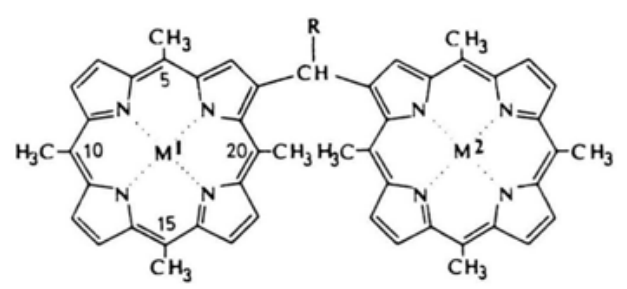

\begin{tabular}{c|ccc}
$\mathbf{3}$ & $\mathrm{M}^{1}$ & $\mathrm{M}^{2}$ & $\mathrm{R}$ \\
\hline $\mathbf{a}$ & $2 \mathrm{H}$ & $2 \mathrm{H}$ & $-\mathrm{H}$ \\
$\mathbf{b}$ & $\mathrm{Ni}$ & $\mathrm{Ni}$ & $-\mathrm{H}$ \\
$\mathbf{c}$ & $2 \mathrm{H}$ & $\mathrm{Ni}$ & $-\mathrm{H}$ \\
$\mathbf{d}$ & $2 \mathrm{H}$ & $2 \mathrm{H}$ & $-\mathrm{CH}_{3}$ \\
$\mathbf{c}$ & $\mathrm{Ni}$ & $\mathrm{Ni}$ & $-\mathrm{CH}_{3}$ \\
$\mathbf{f}$ & $2 \mathrm{H}$ & $\mathrm{Ni}$ & $-\mathrm{CH}_{3}$ \\
$\mathbf{g}$ & $2 \mathrm{H}$ & $2 \mathrm{H}$ & - \\
$\mathbf{h}$ & $\mathrm{Ni}$ & $\mathrm{Ni}$ & - \\
$\mathbf{i}$ & $2 \mathrm{H}$ & $\mathrm{Ni}$ & -
\end{tabular}

Schema 1

Ist an der Methylenbrücke eine Methyl- (3 e) bzw. eine Phenylgruppe ( $\mathbf{3}$ h), so erscheint die bathochrome Teilbande deutlich. Bei den monokomplexierten Dimeren $\mathbf{3} \mathbf{c}$ und $\mathbf{3} \mathbf{f}$ ist dies besonders gut zu erkennen. Durch die Einführung einer Methylgruppe an der Brücke (3f) wird die fast symmetrische SoretBande von 3c stark unsymmetrisch (s. Abbildung 2). Dies sollte eher auf konformative als auf induktive 
Einflüsse der Methylgruppe zurückzuführen sein, worauf weiter unten noch genauer eingegangen werden soll.

In den Neutralbasen $\mathbf{3} \mathbf{a}, \mathbf{3} \mathbf{d}$ und $\mathbf{3} \mathbf{g}$ nimmt die Intensität der bathochromen Teilbande deutlich ab. In der unsymmetrischen Base $\mathbf{2} \mathbf{a}$ ist sie deutlich größer als die der hypsochromen Teilbande. Trotz dieser feinen Unterschiede ist aber die gute Übereinstimmung der Gesamtform der Soret-Bande des unsymmetrischen Diporphinylmethans 2a mit der des symmetrischen 3 a erstaunlich. Dies trifft auch für die Banden der protonierten Formen zu.

Während die Form der Soret-Bande des vierwertigen Kations 2 a offensichtlich nur gering durch die Art des Lösungsmittels beeinflußt wird, beobachtet man für die $\beta-\beta^{\prime}$-verbundenen Dimeren der Struktur 3 in verschiedenen Lösungsmittelgemischen stärker unterschiedliche Spektren.

Deren Veränderung läßt sich nicht mehr nur auf eine Vergrößerung des Kopplungsfaktors zurückführen - wodurch sich nur die Differenz zwischen den Teilbanden nicht aber das Verhältnis der Intensitäten ändert - sondern es kann nur durch eine zusätzliche Konformationsänderung erklärt werden, die eine Veränderung der Internsitätsverhältnisse der Teilbanden hervorruft. Hierauf wird weiter unten noch genauer eingegangen. Als Lösungsmittel diente: Chloroform mit steigender Konzentration an Trifluoressigsäure; konzentrierte $\mathrm{H}_{2} \mathrm{SO}_{4}$; ein Gemisch aus Chloroform/Ethanol (1:1 Volumenteile) angesäuert mit konz. Salzsäure bzw. mit halbkonzentrierter $\mathrm{H}_{2} \mathrm{SO}_{4}$; und die Chloroformphase gesättigt mit konz. $\mathrm{H}_{2} \mathrm{SO}_{4}$, die man beim Schütteln einer Chloroformlösung der dimeren Basen mit konzentrierter $\mathrm{H}_{2} \mathrm{SO}_{4}$ erhält.

Im sichtbaren Spektralbereich zeigen die Banden der dimeren Porphinderivate $\mathbf{2} \mathbf{a}-\mathbf{c}$ und $\mathbf{3} \mathbf{a}-\mathbf{h}$ keine wesentliche Veränderung ihres Musters gegenüber denen der monomeren Porphine $\mathbf{1} \mathbf{a}-\mathbf{g}$. Es ist lediglich eine geringe bathochrome Verschiebung von etwa $3 \mathrm{~nm}$ gegenüber $\mathbf{1}$ a zu beobachten. Dieser Effekt ist aber von der gleichen Größenordnung, wie er auch durch die $\beta$-ständige Diphenylmethylgruppe in $\mathbf{1} \mathbf{f}$ hervorgerufen wird. Eine Aufspaltung tritt nicht ein, wohl aber eine geringe Verbreiterung der Banden. Die Intensität dieser Banden im sichtbaren Spektralbereich ist bei den Dimeren die Summe der beiden einzelnen Porphinringe. Dies ist besonders deutlich bei den mono-metallierten $\mathbf{3 ~ c}$ und $\mathbf{3} \mathbf{f}$ zu erkennen, die in Chloroformlösung mit
Trifluoressigsäure unter Erhalt des Metallkomplexes des einen Ringes am benachbarten protoniert werden können (Abb. 2 u. 3). In einer Kurvenanalyse erhält man durch Superposition der Banden des Nickelkomplexes $\mathbf{1} \mathbf{b}$ und der neutralen Porphinbase 1 a die Kurvenform der Absorptionsbanden von 3 c und $\mathbf{3} \mathbf{f}$ (Abbildung 3 ).

Es sei nebenbei erwähnt, daß besonders die Proben der mono-metallierten Dimeren und der dimeren freien Basen möglichst unter Ausschluß von Licht hergestellt werden. Es zeigte sich, daß diese besonders während der Dünnschichtchromatographie darauf empfindlich durch Zersetzung reagieren. Da es sich bei den hier untersuchten aufgespaltenen Soret-Banden um die Superposition verschieden intensiver Teilbanden handelt, macht sich schon eine geringe Verunreinigung deutlich im Intensitätsverhältnis der Teilbanden bemerkbar. Die Zersetzung der dimeren Porphine sollte zuerst zur Zerstörung nur eines der beiden individuellen Chromophore führen, woraus eine unaufgespaltene Soret-Bande mit einer Bandenlage entsprechend $i_{\text {mono }}$ resultiert, weil eine Excitonenkopplung mit dem benachbarten zersetzten Porphinsystem nicht mehr möglich ist.

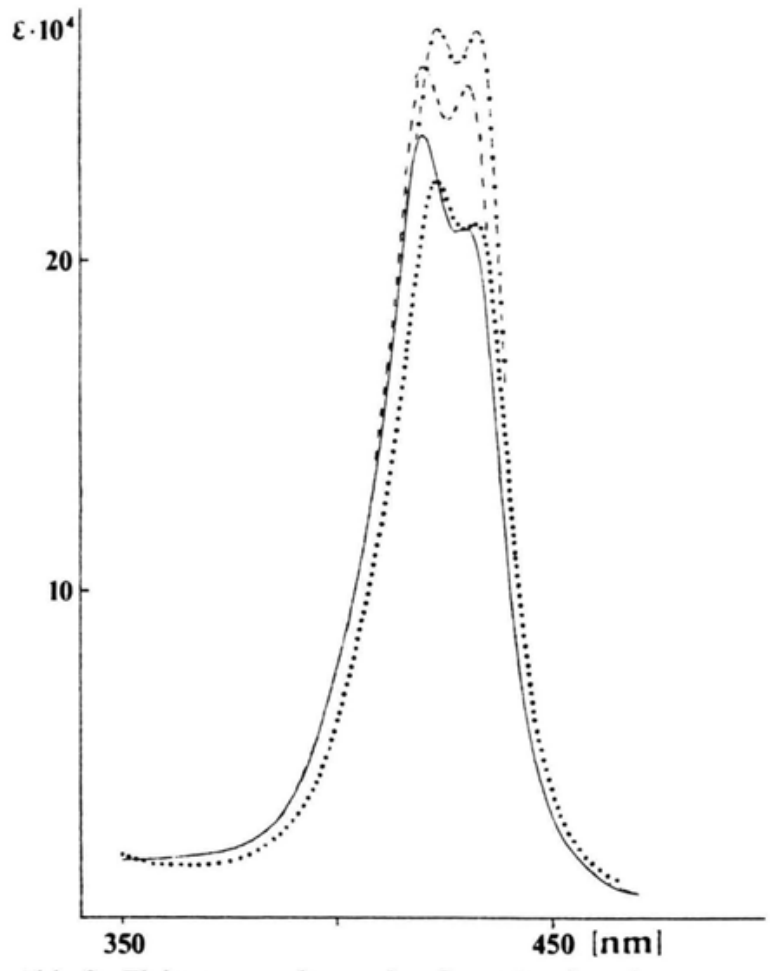

Abb. 2. Elektronenspektren der Soret-Banden der monometallierten Diporphinylmethane 3c $(---), 3 \mathbf{f}(-)$ in $\mathrm{CHCl}_{3}$ und den Dikationen von $3 \mathrm{c}(\cdot-\cdot-\cdot)$ und $\mathbf{3} \mathbf{f}$ $(\cdots \cdots)$ in $\mathrm{CHCl}_{3} / 2 \%$ Trifluoressigsäure. 


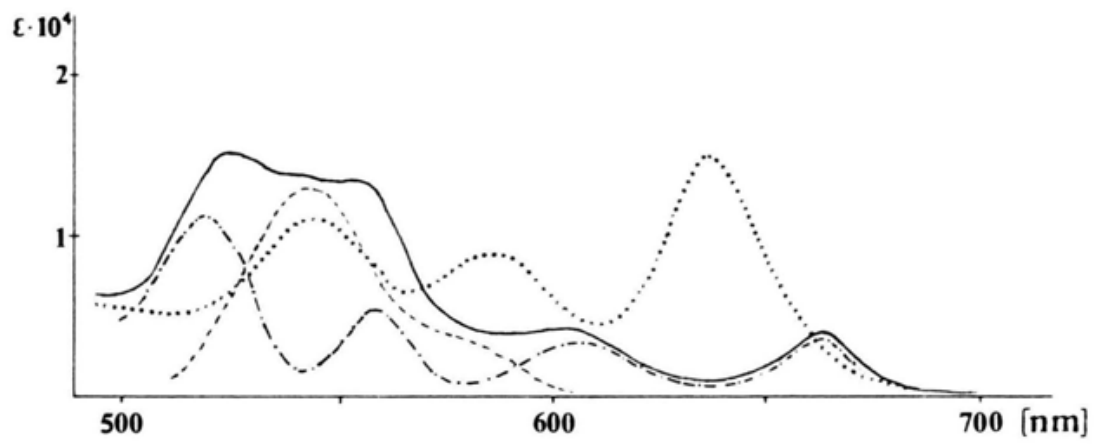

Abb. 3. Elektronenspektrum im sichtbaren Bereich des monometallierten Diporphinylethans $\mathbf{3} \mathbf{f}$ $(-)$ in $\mathrm{CHCl}_{3}$ und seines Kations $(\cdots)$ in $\mathrm{CHCl}_{3} / 2 \%$ Trifluoressigsäure; die ausgezogene Linie ist gleichzeitig die Superposition der abgebildeten Bandenformen des Neutralbasenteiles $(\cdot-\cdot-\cdot)$ und des Nickelkomplexteiles (-...-).

\section{Deutung der Spektren}

Betrachtet man die Spektren der Dimeren 2a-c und $3 \mathbf{a}-\mathbf{h}$ so fällt sofort auf, daß nur die SoretBande (B-Bande) nicht aber die intensitätsschwachen Q-Banden eine wesentliche Veränderung gegenüber denen der monomeren Vergleichssubstanzen zeigen. Weder werden die Q-Banden der zweifach metallierten Komplexe, noch der freien Basen, noch ihrer vierwertigen Kationen durch die Dimerisierung wesentlich im Verhältnis zu den entsprechenden Monomeren verändert. Man kann dies dahingehend interpretieren, da $\beta$ das Energieniveau $\mathrm{e}_{\mathrm{g}}$ (LUMO) hierdurch nicht so berührt wird, daß eine Aufhebung der Entartung in $x$ - und $y$-Komponente erfolgt - außer in dem schon für die monomeren freien Basen diskutierten Rahmen auf Grund der zwei H-Atome im Zentrum des Porphinringes [35-39]. Auch ist eine wesentliche Beeinflussung dieses Niveaus durch eine mögliche Wechselwirkung der Protonen im Innern der beiden benachbarten Porphinringe nicht zu beobachten, selbst nicht im sauren Medium, in dem diese auf Grund der Spektren als vierwertige Kationen vorliegen. Folglich sollte die beobachtete Aufspaltung der SoretBande in zwei Teilbanden auch nicht das Resultat der Aufhebung einer Entartung dieses $\mathrm{a}_{1 \mathrm{u}}-\mathrm{e}_{\mathrm{g}}$ Überganges der einzelnen Chromophorkomponenten im Dimeren sein. Denn es ist nicht einsichtig, warum beim $\mathrm{a}_{1 \mathrm{u}}-\mathrm{e}_{\mathrm{g}}$-Übergang die Entartung durch den benachbarten Ring aufgehoben wird, nicht aber beim $\mathrm{a}_{2 \mathrm{u}}-\mathrm{e}_{\mathrm{g}}$-Übergang der Q-Banden, der besonders empfindlich Veränderungen im Ringsystem anzeigt. Außerdem müßte bei $\beta-\mathrm{CH}_{2}-\beta^{\prime}$-Verknüpfung der Porphine (z.B. 3a) eine andere Q-Bande als bei einer meso- $\mathrm{CH}_{2}-\beta^{\prime}$-Verknüpfung (2 a) resultieren. Bei den Dimeren ist aber kein wesentlicher
Unterschied in den Banden festzustellen (vgl. Abbildung $1 \mathrm{c}$ ).

Auf Grund dieser Argumente ist die beobachtete Aufspaltung der Soret-Bande als Folge einer Excitonenkopplung zwischen diesen Übergangsmomenten der benachbarten Chromophoreinheiten zu deuten. Da diese Kopplung dem Quadrat der Übergangsmomente direkt proportional ist [40], ist auch erklärlich, warum bei den intensitätsschwachen Q-Banden eine Aufspaltung nicht beobachtet wird.

Da diese Dimeren zwangsweise wie Aggregate zu behandeln sind, sollte dafür das von Czikkely, Försterling und Kuhn [31] für Farbstoffaggregate abgeleitete einfach zu handhabende Modell der Wechselwirkung ausgedehnter Dipole befriedigende Ergebnisse liefern.

Danach ist die Anregungsenergie des Farbstoffdimeren $\Delta E^{\prime}$

$$
\Delta E^{\prime} \approx \Delta E \pm 2 J_{1,2}
$$

( $\Delta E$ ist die Anregungsenergie des Monomeren, $J_{1,2}$ das Wechselwirkungsintegral zwischen den Elektronen in den benachbarten Porphinringen).

Der numerische Wert von $J_{1,2}$ ergibt sich zu

$$
J_{1,2}=\frac{\varepsilon^{2}}{D}\left(\frac{1}{a_{1}}+\frac{1}{a_{2}}-\frac{1}{a_{3}}-\frac{1}{a_{4}}\right),
$$

wobei die Übergangsmomente der beiden Farbstoffmoleküle $\mathrm{n}$ und $\mathrm{m}$ durch zwei Dipole mit der Länge $l$ und der Ladung $+\varepsilon$ und $-\varepsilon$ ersetzt wurden.

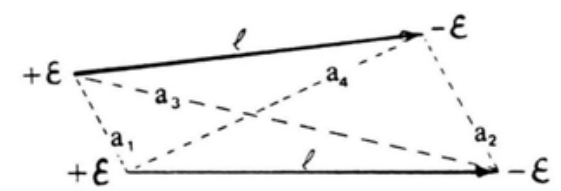

Schema 2 


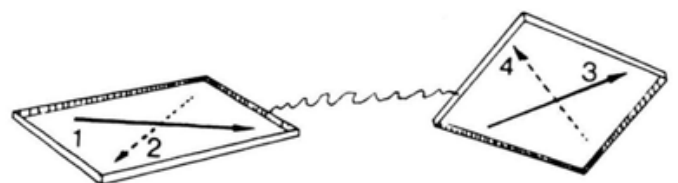

Schema 3

$J_{1,2}$ ist dabei von der Position dieser beiden Dipole zueinander abhängig. Das ,,+"-Zeichen in (1) entspricht bei dieser Stellung einer ,,in-Phase"-Anordnung der Dipole (马), das ,,-“-Zeichen einer ,,gegen-Phase"-Anordnung $(\rightleftarrows)$, analog einer ,inPhase"-Schwingung bzw. ,gegen-Phase"-Schwingung gekoppelter Pendel.

Die Intensitäten der Banden werden analog der Literatur [40] aus der Vereinfachung abgeschätzt, daß sie dem Quadrat der Vektorsummen beider Dipolvektoren proportional sind:

$$
f \approx\left(l_{1} \pm l_{2}\right)^{2} .
$$

Für die Soret-Bande der Porphine werden zwei senkrecht aufeinanderstehende Übergangsmomente verantwortlich gemacht. Deshalb verändert sich diese einfache Wechselwirkung zweier Dipole bei den dimeren Porphinen in die von zwei Paaren gemäß Schema 3. Im Fall der dimeren freien Basen sind zwei tautomere Formen möglich, wie sie für $\mathbf{3}$ a dargestellt sind (für $\mathbf{2}$ a gilt entsprechendes):

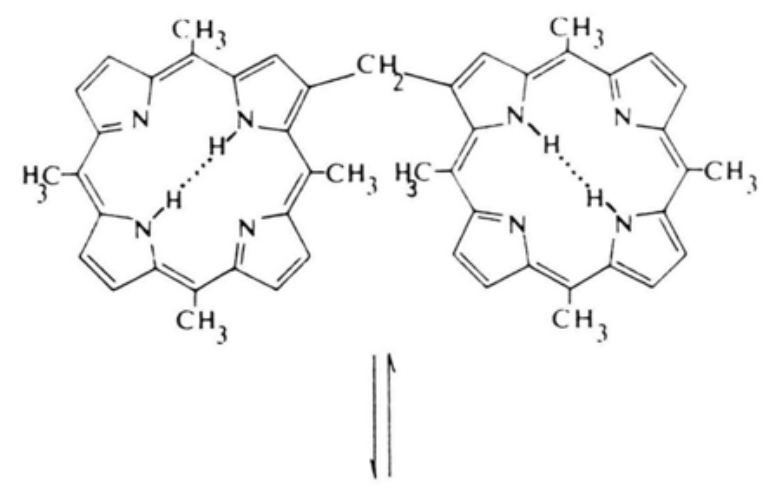

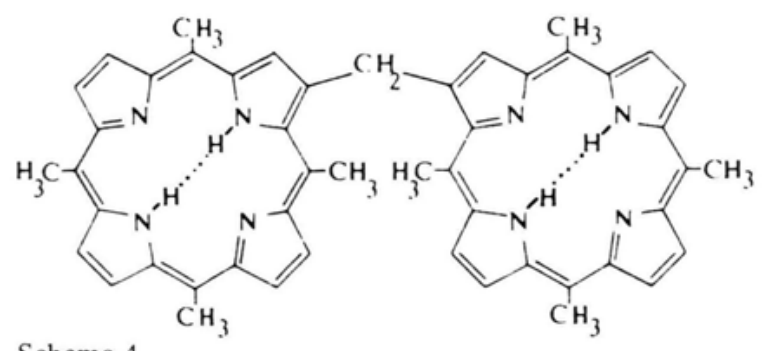

Da bisher über diese tautomeren Formen und deren mögliches Gleichgewicht und den dazugehörigen Übergangsmomenten $B_{x}$ und $B_{y}$ keine Aussage gemacht werden konnte, ist eine Anwendung des Modells der Wechselwirkung ausgedehnter Dipole auf die dimeren freien Basen vorerst nicht möglich.

Bei den vierwertigen Kationen dieser Dimeren sind die Verhältnisse entschieden einfacher und günstiger. Übereinstimmend lieferten alle Rechnungen und theoretischen Betrachtungen für die SoretBande der monomeren Dikationen der Porphine doppelt entartete Elektronenübergänge, die senkrecht zueinander polarisiert sind. Daraus folgt, da $\beta$ die dazugehörigen Übergangsmomente $B_{x}$ und $B_{y}$ gleich groß sind, entsprechend zwei gleichen, senkrecht aufeinanderstehenden Dipolen. Gleiches gilt auch für die dimeren Metallkomplexe. Da sich die Form der beiden Q-Banden auch der dimeren vierwertigen Kationen gegenüber der der monomeren Dikationen nicht verändert hat, ist die Folgerung zulässig, daß die Lage der Protonen im Innern der einzelnen Porphinringe und damit auch die $\mathrm{D}_{4 \mathrm{~h}}$ Symmetrie der individuellen Chromophoreneinheit ungestört blieb. Offensichtlich werden die elektrostatischen Wechselwirkungen auch durch die in Lösung vorhandenen Anionen kompensiert. Aus diesen Gründen werden für die weiteren Betrachtungen die dimeren Metallkomplexe und die vierwertigen Kationen herangezogen:

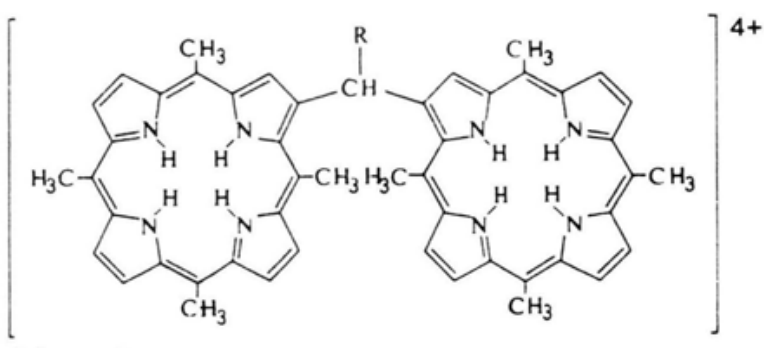

Schema 5

Somit sind die beiden kovalent verbundenen Chromophore durch zwei jeweils senkrecht aufeinanderstehende gleichgroße Dipole $l$ bis 4 (Abb. 4) mit der Länge $l$ und der Ladung $+\varepsilon$ und $-\varepsilon$ zu ersetzen.

Wir denken uns zunächst die beiden Chromophoren getrennt. Wegen der $\mathrm{D}_{4 \mathrm{~h}}$-Symmetrie der monomeren Dikationen ist durch Linearkombination der dazugehörigen Wellenfunktion jede Richtung dieser 


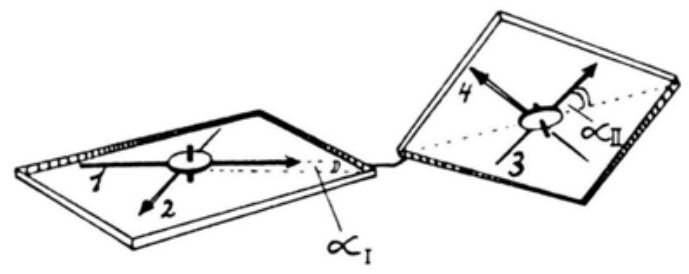

Abb. 4. Modelldarstellung der bei Vernachlässigung der Kopplung um die Winkel $\alpha_{1}$ und $\alpha_{I I}$ drehbaren Dipolpaare im dimeren Porphinderivat, in dem die einzelnen Chromophorkomponenten $\mathrm{D}_{4 \mathrm{~h}}$-Symmetrie besitzen. Durch die Kopplung wird die Entartung aufgehoben, $\alpha_{1}$ und $\alpha_{\text {II }}$ werden festgelegt.

beiden dazugehörigen senkrecht aufeinanderstehenden Übergangsmomente möglich, d.h. $\alpha_{I}$ und $\alpha_{I I}$ sind nicht festgelegt [41]. Nähert man zwei dieser Systeme einander, so wird die Entartung aufgehoben. Man findet die Anregungsenergie $\Delta E$, indem $\operatorname{man} \alpha_{I}$ und $\alpha_{\text {II }}$ solange verändert, bis die Wechselwirkung von Dipol 1 mit 4 und 2 mit 3 aufgehoben ist. Dann berechnet man für die vier gekoppelten Schwingungen die Anregungsenergien nach (1) und (2).

Praktisch wurde folgendermaßen vorgegangen: mit Hilfe von Kalottenmodellen wurde zuerst eine sterisch möglichst günstige Stellung der beiden Porphinringe gesucht. Dann wurde unter Beibehaltung dieser Konformation, an einem Dreiding-Modell, das im Zentrum der einzelnen Porphinringe frei drehbare Scheiben mit fest senkrecht zueinander stehenden Dipolen (wie dargestellt) enthielt, die Winkel $\alpha_{1}$ und $\alpha_{\text {II }}$ solange variiert bis der Dipol 1 nach (2) keine Wechselwirkung mehr mit Dipol 4 und gleichzeitig Dipol 2 keine mit Dipol 3 zeigt. Waren diese Positionen gefunden, dann erst wurde die Wechselwirkung von 1 mit 3 und 2 mit 4 nach (6) (s. Anhang) bzw. die dazugehörigen Bandenlagen berechnet. Die dazugehörigen relativen Bandenintensitäten wurden dem Quadrat der Vektorsumme der entsprechenden ,in-Phase“- bzw. ,gegen-Phase"-Anordnung der Dipole 1 und 3 bzw. 2 und 4 gleichgesetzt. Man erhält so vier Bandenlagen und die vier dazugehörigen relativen Intensitäten $\mathrm{f}$.

Wie in der Literatur wird $D=2,5$ gesetzt und die Größen $l$ und $\varepsilon$ werden aus der Absorption eines cyclophanartigen Porphindimeren ausreichend bekannter Geometrie bestimmt (s. Anhang). Es ergeben sich dabei die Werte $l=4,05 \AA, \varepsilon=0,33 e_{0}$.

In Abb. 5 und Abb. 6 sind die dann zu erwartenden Bandenverschiebungen für verschiedene Anord- nungen eines Dipolpaares angegeben. Danach ergibt sich für nebeneinander liegende Dipole eine bathochrome, für parallel übereinander liegende eine hypsochrome Verschiebung der Teilbanden, die mit kleiner werdendem Abstand größer wird. Für rechtwinklig zueinanderstehende Dipole wird eine Aufspaltung erwartet, wobei beide Teilbanden gleiche Intensität aufweisen. Ist der Winkel zwischen den beiden Dipolen größer $90^{\circ}$, ist die bathochrome, ist er kleiner $90^{\circ}$, ist die hypsochrome Teilbande intensiver; geringfügige Abweichungen vom rechten Winkel führen sofort zu sichtbaren Veränderungen der Intensitätsverhältnisse. Bei cyclophanartiger und auch bei nebeneinander liegender Anordnung der Porphinringe ist eine Veränderung der Soret-Bande auf Grund der Kopplung kaum noch feststellbar, wenn der Mittelpunktsabstand größer als $\sim 15 \AA$ ist.

Allerdings ist hierbei zu beachten, daß nach (4) (Anhang) die Dipollänge $l$ und damit auch $\varepsilon$ von den Größen $M, J_{1,2}, a_{1}$, wie auch $D$ abhängig sind. Eine Fehlerbreite von $+10 \%$ bis $-10 \%$ dieser GröBen führt für $M$ zu einer Schwankung von $l$ in dem Bereich von $+37 \%$ bzw. $-37 \%$, für $J_{1,2}$ von $-16 \%$ bis $+16 \%$, für $a_{1}$ von $-49 \%$ bis $+34 \%$ und für $D$ von $-16 \%$ bis $+17 \%$. Deshalb sind im Anhang zwei weitere Beispiele für die Berechnung von $l$ gegeben, bei denen die Größen $M, J_{1,2}, a_{1}$ und $D$ innerhalb der

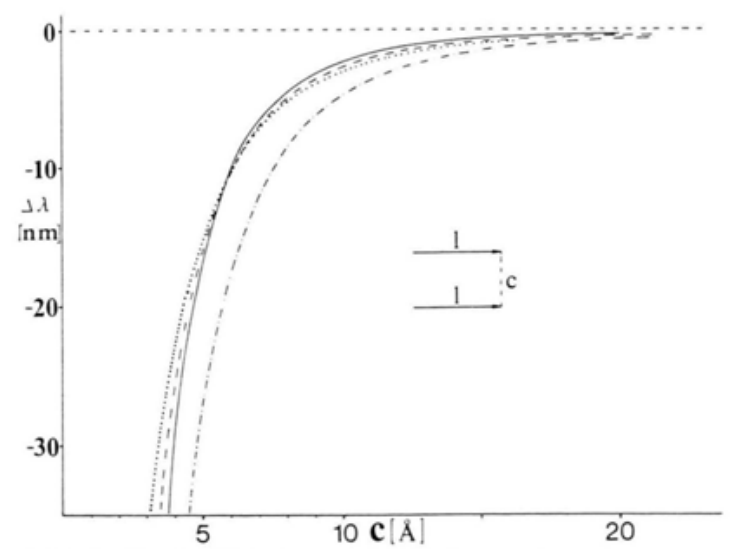

Abb. 5. Nach Gleichung (6) (Anhang) berechnete hypsochrome Bandenverschiebung $\left(\Delta \lambda=\lambda_{\text {dimer }}-\lambda_{\text {mono }}\right)$ für parallel exakt übereinander ,in-Phase“ angeordnete Dipole in Abhängigkeit des Abstandes $c$ und verschiedener Dipollängen $l$ (Beispiel a und $b$, Anhang, Erklärung im Text) für cyclophanartige vierwertige Kationen $\left(\lambda_{\text {mono }}=428 \mathrm{~nm}\right)$ : $(---): l=4,05 \AA, k=1,0 ;(\cdot-\cdot-\cdot): l=4,05, k=1,75$; $(\cdots): l=5,6 \AA, k=1,0$ (Beispiel a); $(\square): l=2,2 \AA$, $k=1,0$ (Beispiel b). 


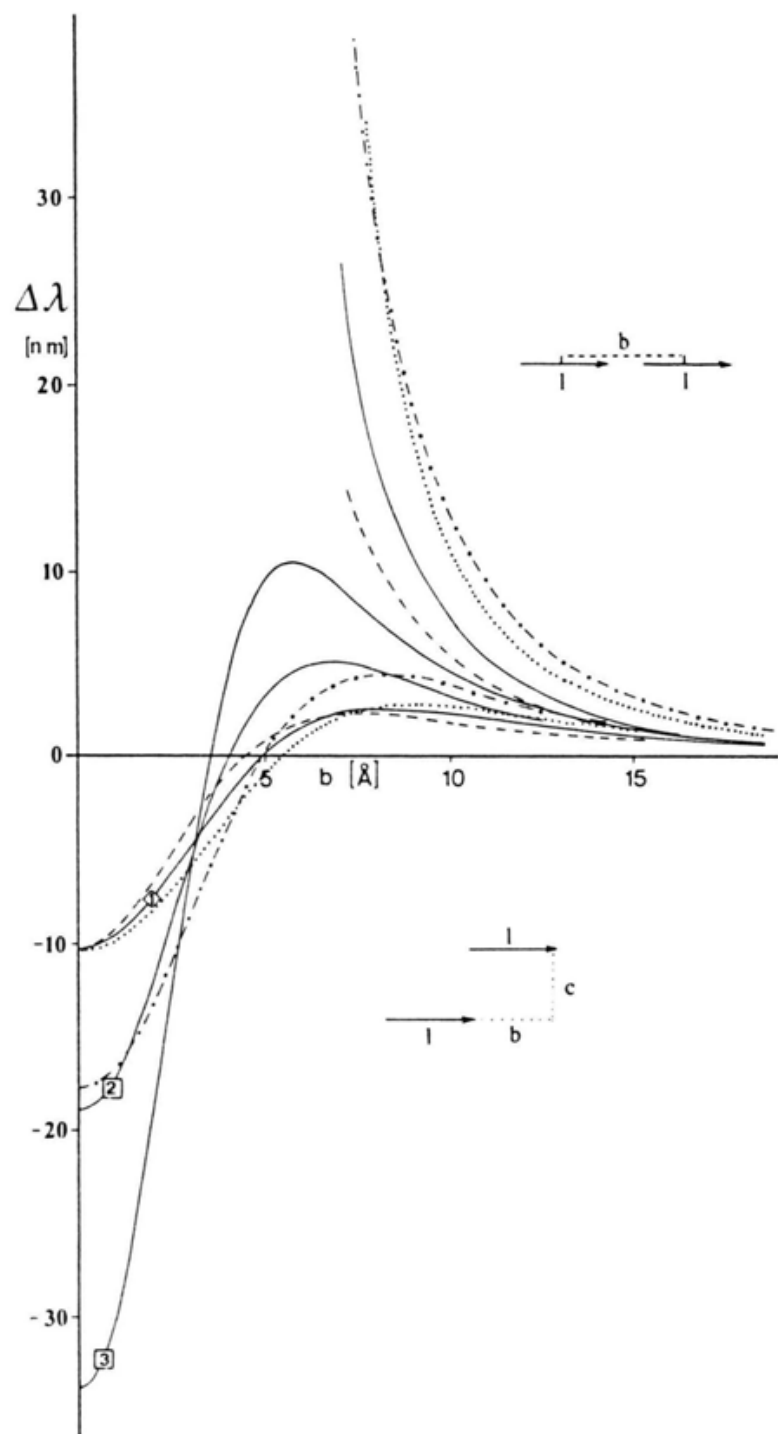

Abb. 6. Nach Gleichung (6) berechnete Bandenverschiebungen für parallel ,,in-Phase" angeordnete Dipole in Abhängigkeit der Verschiebung b und des Abstandes $c$. Oben: für exakt hintereinander liegende Dipole: $(-): l=4,05 \AA$, $k=1,0 ;(\cdot-\cdot-\cdot): l=4,05 \AA, k=1,75 ;(\cdots): l=5,6 \AA$, $k=1,0$ (Beispiel a, Anhang); (----): $l=2,2 \AA, k=1,0$ (Beispiel b). Unten: für versetzt parallel angeordnete Dipole: Fall $1 c=6 \AA: l=4,05 \AA, k=1,0 ;(\cdot-\cdot-\cdot): l=4,05 \AA$, $k=1,75 ;(\cdots): l=5,6 \AA, k=1,0$ (Beispiel a); (----): $l=2,2 \AA, k=1,0$ (Beispiel b); Fall $2 c=4,5 \AA$ $l=4,05 \AA, k=1,0 ;$ Fall $3 c=3,5 \AA:(-): l=4,05 \AA$, $k=1,0$.

angegebenen Fehlerbreite liegen und für die sich ein sehr kurzer Dipol $(l=2,2 \AA)$ und ein längerer $(l=5,61 \AA)$ errechnet. Wie man den Abbn. 5 und 6 entnehmen kann, ergibt sich für parallel übereinanderliegende Dipole eine etwa gleichgroße Verschie- bung der Teilbande trotz verschiedener Dipollängen und den dazugehörigen $\varepsilon^{2} / D$-Werten. Erst bei nebeneinanderliegender Anordnung zeigt sich mit größerer Dipollänge eine stärkere Verschiebung der Teilbanden.

Hinzu kommt, daß das zwischen den beiden wechselwirkenden Chromophoren liegende Medium einen Einfluß auf die Aufspaltung ausüben sollte. So ist bei Anthracenophanen, wie auch Naphthalenophanen, die über [2.2]-Brücken sehr dicht parallel zueinander angeordnet sind, eine deutlich geringere hypsochrome Verschiebung von $\lambda_{\text {dimer }} \mathrm{zu}$ beobachten als man theoretisch erwartet und wie man für die Aufspaltung mehr schräg nebeneinanderliegender Chromophore im Einklang mit der Beobachtung ableiten kann [32].

Die hier genannten Fehlermöglichkeiten beeinflussen über $l$ bzw. $\varepsilon^{2} / D$ lediglich die Größe der Aufspaltung der Teilbanden, während sich die Stellung der Dipole zueinander bzw. die Winkel $\alpha_{1}$ und $\alpha_{\text {II }}$ und damit die Intensitäten der Teilbanden nicht nennenswert ändert. Um dem möglichen Einfluß dieser Fehler gerecht zu werden, wurde vereinfachend $\pm 2 \varepsilon^{2} / D$ mit einem Korrekturfaktor $k$ versehen und für die Berechnung (6) (Anhang) mit $k\left( \pm 2 \varepsilon^{2} / D\right)$ verwendet. Durch Variation von $k$ zwischen 1 und 2,5 in 0,25-Schritten wurde eine optimale Anpassung der berechneten Aufspaltung einer untersuchten Konformationsmöglichkeit an die beobachtete Soret-Bande zu erreichen versucht. Für alle hier untersuchten, nebeneinander angeordneten Porphinringe ergab sich die beste Übereinstimmung, wenn mit $k=1,75$ gerechnet wurde. Dies sollte auch zulässig sein, da diese Korrektur in seiner Wirkung einer Verlängerung des ausgedehnten Dipols auf $l=5,6 \AA$ und einer Veränderung von $D=2,5$ auf $D=2,25$ äquivalent ist und damit deutlich innerhalb der angegebenen Fehlergrenzen liegt. Für cyclophanartige Porphindimere ergab sich eine gute Übereinstimmung mit dem unkorrigierten Wert $(k=1)$. Dies ist nach Abb. 5 auch verständlich, da bei dieser Anordnung der Dipole nur eine geringe Abhängigkeit von deren Länge vorliegt.

Um Soret-Banden nach den in Abb. 5 und 6 dargestellten Verschiebungen zu beobachten, ist aber auch eine stark bevorzugte Konformation der dimeren Porphine erforderlich. Sind mehrere möglich, so resultiert eine Überlagerung verschiedener Bandenformen, die eine Interpretation erheblich erschweren. 


\section{Vierwertiges Kation des Dimeren 2}

Bei den hier diskutierten Dimeren der Struktur 2 konnte an Hand von Kalottenmodellen und mit Hilfe ${ }^{1} \mathrm{H}-\mathrm{NMR}$-spektroskopischer Messungen gezeigt werden, daß die Möglichkeit verschiedener Konformationen stark eingeschränkt ist und in Lösung die in Abb. 7 symbolisierte bevorzugt vorliegen sollte [23]. Dies müßte sich auch durch die Veränderung der Soret-Bande nachweisen lassen, wie sie durch die oben beschriebene Excitonenkopplung hervorgerufen wird. Danach koppelt der Dipol 2 weder mit 4 noch mit 3, da er zu diesen orthogonal steht. Dies ergibt eine unverschobene Teilbande bei $428 \mathrm{~nm}$. Diese Wellenlänge entspricht dem Einfluß eines schweren Substituenten sowohl in meso- als auch in $\beta$-Position eines vergleichbaren monomeren zweiwertigen Kations, betrachtet man die Lage der Soret-Banden der Dikationen von 1 a $(421 \mathrm{~nm}), 1$ d $(421 \mathrm{~nm})$ und $1 \mathrm{f}(427 \mathrm{~nm})$ beziehungsweise die Verschiebungstendenzen der Metallkomplexe $\mathbf{1 b}, \mathbf{1}$ e, $1 \mathrm{~g}$ und $\mathbf{1 h}$. Nach der Ausgangsbedingung koppelt Dipol 4 nicht mit 1 . Eine intensive Kopplung ergibt sich zwischen den Dipolen 1 und 3. Nach den aus dem Modell bestimmten Abständen $a_{1}, a_{2}, a_{3}$ und $a_{4}$ errechnet sich nach (2) und (1) für die ,inPhase"-Anordnung eine um $13 \mathrm{~nm}$ bathochromver-

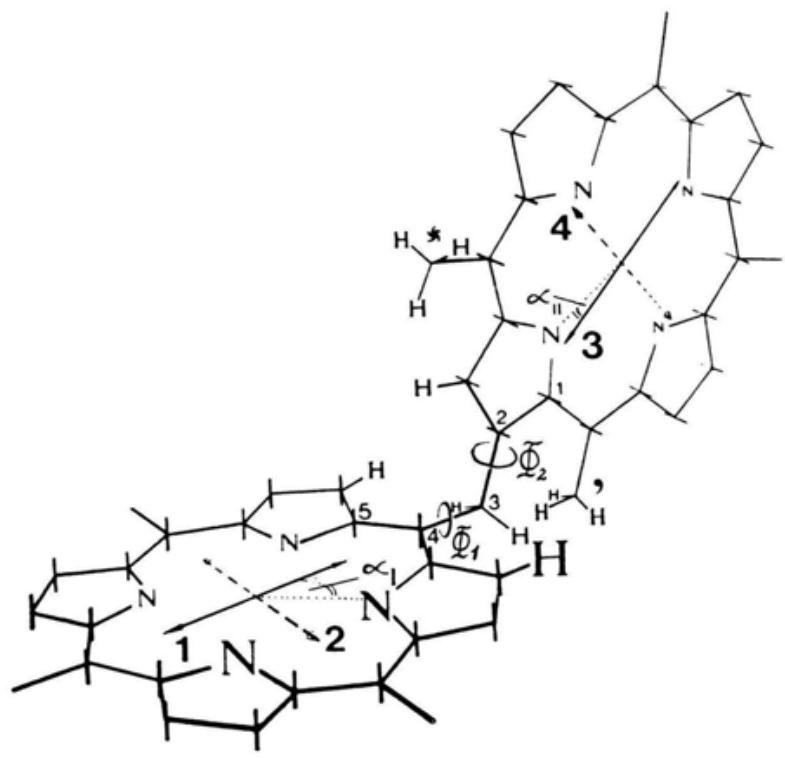

Abb. 7. Stellung der Dipole mit größtmöglicher Wechselwirkung im vierwertigen Kation des Dimeren $\mathbf{2}$ a in sterisch günstiger Konformation mit $\Phi_{1}=90^{\circ}$ und $\Phi_{2}=180^{\circ}$ $\left(\Phi_{1}\right.$ ist definiert durch die Atome $\mathrm{C}_{2}-\mathrm{C}_{3}-\mathrm{C}_{4}-\mathrm{C}_{5}$ und $\Phi_{2}$ durch $\left.\mathrm{C}_{1}-\mathrm{C}_{2}-\mathrm{C}_{3}-\mathrm{C}_{4}\right) ; \alpha_{1}=45^{\circ}, \alpha_{\mathrm{II}}=10^{\circ}$. schobene Teilbande $(441 \mathrm{~nm})$ und für die ,gegenPhase"-Anordnung eine um $12 \mathrm{~nm}$ hypsochrom verschobene $(416 \mathrm{~nm})$. Die dazugehörigen relativen Bandenintensitäten $f$ werden dem Quadrat der entsprechenden Vektorsumme der koppelnden Dipole gleichgesetzt.

Auf Grund dieser berechneten Bandenlagen wurden Kurven mit der Halbwertsbreite $(\sim 13 \mathrm{~nm})$ der Soret-Bande des monomeren zweiwertigen Kations von $1 \mathbf{f}$ und der berechneten Bandenintensität $f$ gezeichnet. Die Superposition dieser ergibt den in Abb. 8 a dargestellten Kurvenverlauf der so berechneten Soret-Bande des vierwertigen Kations von 2 a mit der in Abb. 7 dargestellten Konformation.

Es zeigt sich eine sehr gute Übereinstimmung zwischen experimenteller (Abbildung $1 \mathrm{c}$ und $8 \mathrm{e}$ ) und nach diesem Modell abgeleiteter Soret-Bande (Abbildung $8 \mathrm{a}$ ). Trotzdem liegt die beobachtete hypsochrome Teilbande $(425 \mathrm{~nm}$, vgl. Tab. 1) noch deutlich gegenüber der bei $428 \mathrm{~nm}$ angenommenen Bande eines entsprechenden Monomeren nach kürzeren Wellenlängen verschoben.

Um den Einfluß der Konformation auf den Verlauf der Soret-Bande und auch deren Aussagekraft $\mathrm{zu}$ verdeutlichen, seien einige weitere dargestellt (vgl. Abbildung 8). Verändert man die Konformation, $\left(\Phi_{1}=90^{\circ}, \Phi_{2}=180^{\circ}\right)$ in der die beiden Ebenen exakt senkrecht zueinander stehen, geringfügig dahingehend, daß $\Phi_{2}= \pm 150^{\circ}$ wird, so ergibt sich immer noch eine gute Übereinstimmung. Und zwar tritt nun auch eine Kopplung zwischen den Dipolen 2 und 4 ein, die für die ,in-Phase“-Anordnung zu einer geringfügig hypsochrom verschobenen intensiveren Bande bei $426 \mathrm{~nm}$ und für die, ,gegenPhase"-Anordnung zu einer schwächeren bei $430 \mathrm{~nm}$ führt. Die Kopplung von $1-3$ führt zu einer intensiven Teilbande bei $440 \mathrm{~nm}$ und einer schwachen bei $417 \mathrm{~nm}$. Eine entsprechende Superposition ergibt die in Abb. $8 \mathrm{~b}$ dargestellte Bandenform. Diese Konformation könnte durch die sterische Wechselwirkung der meso-ständigen Methylgruppe mit der benachbarten Brücken-Methylengruppe hervorgerufen werden, wie es in Abb. 9 dargestellt ist. Danach ist die Stellung B der H-Atome energetisch günstiger. Und bedenkt man, daß ein Energiegewinn von nur $2 \mathrm{kcal} / \mathrm{mol}$ ausreichend ist, um diese Konformation erheblich zu bevorzugen, so scheint dies nicht überinterpretiert zu sein.

Führt man eine weitergehende Konformationsänderung durch, so daß die beiden Porphinebenen 

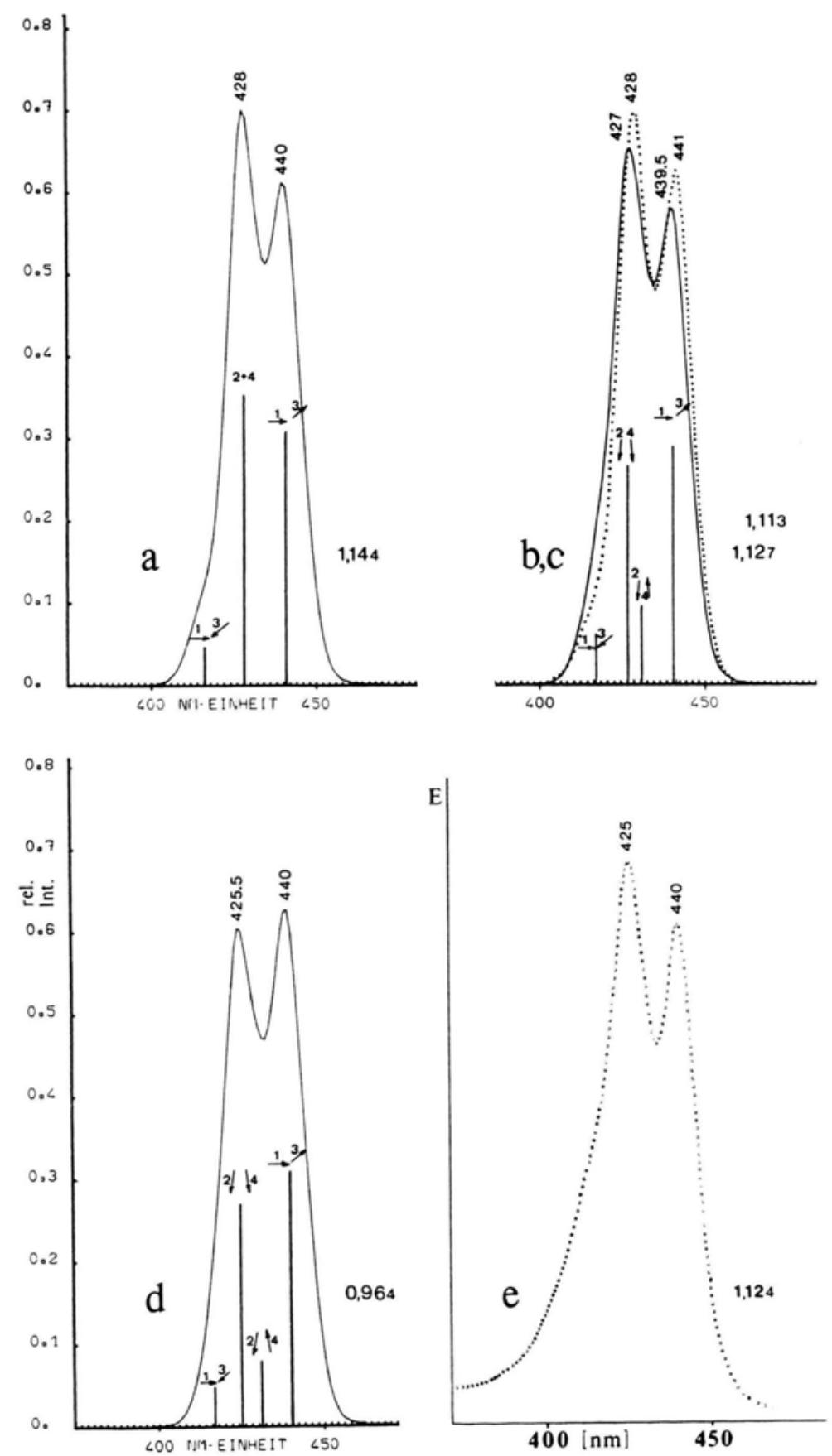

Abb. 8. Aus der Superposition der berechneten Teilbanden erhaltene SoretBanden des vierwertigen Kations $\mathbf{2}$ a in Abhängigkeit von $\Phi_{1}$ und $\Phi_{2}$. Eingezeichnet sind die berechneten Lagen der ,in- und gegen-Phase"-Anordnung der Dipolpaare $1-3$ und $2-4$, die Höhe ist der relativen Oszillatorenstärke f proportional. Die Zahlen neben den Kurven geben das relative Extinktionsverhältnis $E_{\text {hypso }} / E_{\text {batho }}$ an. $\left(\right.$ a: $\Phi_{1}=90^{\circ}, \Phi_{2}=180^{\circ}$, $\alpha_{1}=45^{\circ}, \alpha_{\text {II }}=10^{\circ} ;$ b: $\Phi_{1}=90^{\circ}, \Phi_{2}=$ $-150^{\circ}, \alpha_{1}=45^{\circ}, \alpha_{\mathrm{II}}=12.5^{\circ} ;$ c $(\cdots)$ : $\Phi_{1}=75^{\circ}, \Phi_{2}=-160^{\circ}, \alpha_{\mathrm{I}}=52^{\circ}, \alpha_{\mathrm{II}}=$ $-8^{\circ}$ (für b sind die berechneten Lagen mit $\mathrm{f}$ eingezeichnet); $\mathrm{d}: \Phi_{1}=75^{\circ}, \Phi_{2}=$ $160^{\circ}, \alpha_{1}=47^{\circ}, \alpha_{I I}=12^{\circ}$; e: experimentell.)

in einer aufgeklappten Form zueinander stehen $\left(\Phi_{1}=75^{\circ}, \Phi_{2}=160^{\circ}\right)$, so tritt eine deutliche Veränderung der abgeleiteten Soret-Bande ein (vgl. Abb. $8 \mathrm{~d}$ ), die nicht mehr mit der beobachteten im Einklang ist (vgl. Abbildung $8 \mathrm{e}$ ).
Zur Überprüfung dieser qualitativen Aussage wurde zusätzlich eine Konformations-Energie-Berechnung durchgeführt [42]. Als Grundlage für die Bindungslängen und Winkel des Porphinringes wurden die kristallographisch ermittelten Werte des Ni- 
TMP 1 b, des Nickel-octaethylporphins $[43,44]$ und des Kupfer-meso-Tetra-n-propylporphins [45] herangezogen (vgl. Angaben in Tabelle 2). Die Porphinringe wurden dabei als vollständig planar angenommen. Variiert man die Torsionswinkel $\Phi_{1}$ und $\Phi_{2}$ in $20^{\circ}$-Schritten und führt gleichzeitig eine Energie-Minimalisierung der zur Methylenbrücke benachbarten meso-ständigen Methylgruppe um ihre $\mathrm{C}-\mathrm{C}$-Bindung durch, um deren sterische Behinderung möglichst gering zu halten, so erhält man den in Abb. 10 dargestellten Ramachandran-Plot. Wie schon früher erwähnt [23], zeigt er die starke sterische Behinderung der beiden Porphinringe bei Rotation um die beiden Bindungen der $\mathrm{CH}_{2}$ Brücke. Da in dieser Rechnung nur die Torsionswinkel, nicht aber auch gleichzeitig die Bindungswinkel oder -längen variiert werden konnten, und die meso-Methylgruppe in $5^{\prime}$-Position eine starke Wechselwirkung zur Methylenbrücke aufweist, erscheint der Bereich der energetisch möglichen Konformation sehr klein. Deshalb sind auch die isoEnergiekurven eingetragen, die noch $200 \mathrm{kcal} / \mathrm{mol}$ über der energetisch günstigsten Konformation liegen. Denn es konnte gezeigt werden, daß geringfügige Bindungswinkelveränderungen von nur $2^{\circ}$ zum Teil erhebliche Energiegewinne bringen, da die sterische Behinderung sofort erheblich nachlassen kann. Das Wesentliche war aber, daß die auf Grund der Elektronenspektren oben gemachte Aussage bestätigt wurde, wonach die beiden Porphinebenen in der energetisch günstigsten Konformation nicht exakt senkrecht $\left(\Phi_{1}= \pm 90^{\circ} ; \Phi_{2}= \pm 180^{\circ}\right)$ zueinanderstehen, sondern leicht zueinander geneigt sein sollten $\left(\Phi_{1} \approx 75^{\circ} ; \Phi_{2} \approx-165^{\circ}\right.$ bzw. $\Phi_{1} \approx 105^{\circ}$; $\Phi_{2} \approx 165^{\circ}$ ). Wie in Tab. 2 gezeigt, ist diese Energiedifferenz nur sehr gering $(0,3-2,1 \mathrm{kcal} / \mathrm{mol})$ und auch von den Bindungswinkeln abhängig, sie zeigt aber für die Bindungswinkel mit möglichst geringer sterischer Behinderung den größten Wert, d. h. auch die größte Abweichung von der senkrechten Stellung der beiden Ringe zueinander (Tab. $2 \mathrm{f}$ u. g). Die zu dieser Konformation gehörige berechnete Soret-Bande gleicht denen in Abbildung 8 b, c. Sowohl das Intensitätsverhältnis der beiden Teilbanden, wie auch die deutlich erkennbare hypsochrome Schulter der kurzwelligen Bande zeigen eine sehr gute Übereinstimmung mit der beobachteten SoretBande des vierwertigen Kations von 2 a. Betrachtet man den Kurvenverlauf der exakt senkrecht zueinander stehenden Porphinebenen $\Phi_{1}=90^{\circ}$, $\Phi_{2}=180^{\circ}$ und der am stärksten ,abgekippten“" (Abb. 8c) Anordnung $\left(\Phi_{1}=75^{\circ}, \Phi_{2}=-160^{\circ}\right)$, so

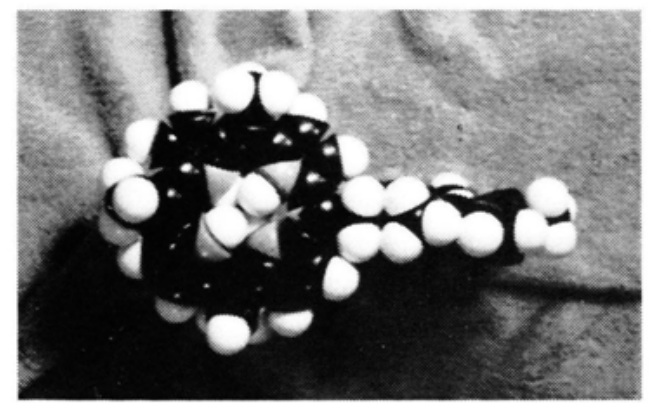

A

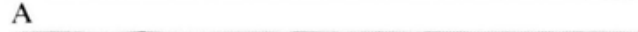

Abb. 9. Sterische Wechselwirkung der mesoständigen Methylgruppe in 5'-Position mit der benachbarten Methylenbrücke im Dimeren 2: im Vergleich zu Abb. 7 wird das Molekül von unten betrachtet. $\mathrm{A}=$ energetisch ungünstiger, $\mathrm{B}=$ günstiger (vgl. Tabelle 2$)$.

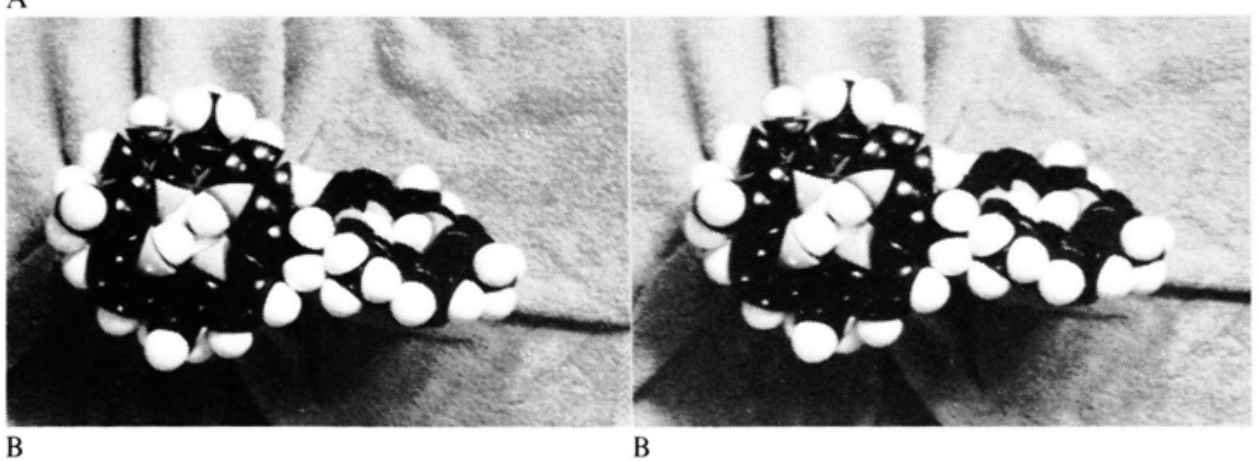


Tab. 2. Einfluß verschiedener Bindungslängen und Winkel auf die Abweichung von der exakt senkrechten Anordnung der Porphinebenen in $\mathbf{2}$ im Energieminimum.

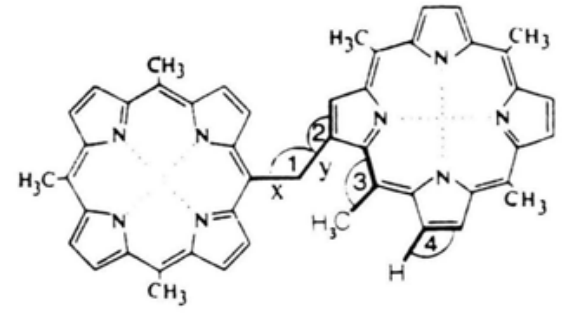

\begin{tabular}{lllllllllll}
\hline & $x$ & $y$ & 1 & 2 & 3 & 4 & $\Phi_{1}$ & $\Phi_{2}$ & $\Delta E_{\mathrm{tot}_{1}} \Delta E_{\mathrm{tot}_{2}}$ \\
\hline a) & 1,518 & 1,518 & $109,5^{\circ}$ & $125^{\circ}$ & $117,5^{\circ}$ & $127,2^{\circ}$ & $81^{\circ}$ & $-177^{\circ}$ & $-0,4 \mathrm{kcal}$ & 0,0 \\
b) & 1,518 & 1,518 & $109,5^{\circ}$ & $127^{\circ}$ & $117,5^{\circ}$ & $127,2^{\circ}$ & $81^{\circ}$ & $-178^{\circ}$ & $-0,4$ & 11,1 \\
c) & 1,518 & 1,518 & $109,5^{\circ}$ & $123^{\circ}$ & $117,5^{\circ}$ & $127,2^{\circ}$ & $81^{\circ}$ & $-175^{\circ}$ & $-0,4$ & $-6,3$ \\
d) & 1,518 & 1,518 & $105^{\circ}$ & $125^{\circ}$ & $117,5^{\circ}$ & $127,2^{\circ}$ & $83^{\circ}$ & $-176^{\circ}$ & $-0,3$ & $-1,8$ \\
e) & 1,518 & 1,518 & $113^{\circ}$ & $125^{\circ}$ & $120^{\circ}$ & $127,2^{\circ}$ & $79^{\circ}$ & $-170^{\circ}$ & $-1,2$ & $-1,2$ \\
f) & 1,525 & 1,525 & $105^{\circ}$ & $123^{\circ}$ & $120^{\circ}$ & $127,2^{\circ}$ & $80^{\circ}$ & $-167^{\circ}$ & $-2,1$ & $-7,0$ \\
g) & 1,525 & 1,525 & $105^{\circ}$ & $123^{\circ}$ & $120^{\circ}$ & $125^{\circ}$ & $81^{\circ}$ & $-167^{\circ}$ & $-1,4$ & $-8,8$ \\
\hline
\end{tabular}

$\Delta E_{\text {tot }_{1}}$ : Energiedifferenz zwischen der minimalisierten und der exakt senkrechten Anordnung $\left(\Phi_{1}=90^{\circ}, \Phi_{2}=180^{\circ}\right)$ mit den veränderten Winkeln $(1,2,3,4)$ und Bindungslängen $(x, y)$.

$\Delta E_{\mathrm{tot}_{2}}$ : Energiedifferenz zwischen der minimalisierten Konformation mit veränderten Bindungslängen und Winkeln und der Konformation a (Grundlage des Ramachandran-Plot in Abbildung 10).

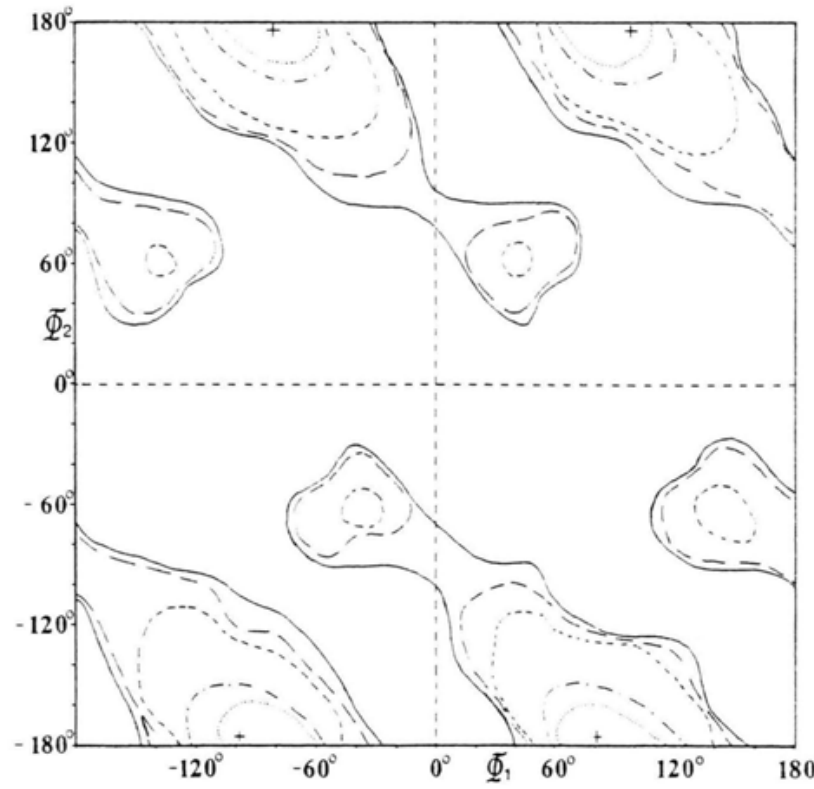

Abb. 10. Die isoenergetischen Kurven der Konformationen des Diporphinylmethans $\mathbf{2}$ a als Funktion der Torsionswinkel $\Phi_{1}$ und $\Phi_{2}$ in $20^{\circ}$-Schritten. Die markierten Positionen zeigen die energetisch günstigsten Konformationen an. $(\cdots)$ ca. $5,0 \mathrm{kcal} \mathrm{mol}, \cdot-\cdot \cdot 10, \cdots 100,---500$ und $1000 \mathrm{kcal} / \mathrm{mol}$ über dem globalen Minimum +.) ist die Differenz nur sehr gering. Es ist aber zu erkennen, da $\beta$ die Berechnung der leicht ,abgekippten" Anordnung ein besseres Intensitätsverhältnis in bezug zum experimentell gefundenen aufweist; auch ist demzufolge die hypsochrome Schulter der kurzwelligen Teilbande deutlicher. Dieser Vergleich deutet die Grenze der Aussagekraft dieser Berechnungen an.

Weiterhin sei darauf hingewiesen, daß die Kopplung der beiden Porphinchromophore auch von der Polarität des Lösungmittels abhängt (Abb. 11). So nimmt die Differenz der hypsochromen zur bathochromen Teilbande in einer $\mathrm{CHCl}_{3} / \mathrm{CF}_{3} \mathrm{COOH}$-Lösung mit steigener Konzentration an $\mathrm{CF}_{3} \mathrm{COOH}$ deutlich zu. Nimmt man beim Versetzen einer Chloroformlösung 2 a mit $\mathrm{CF}_{3} \mathrm{COOH}$ den Kurvenverlauf, wo im Bereich der Q-Banden nur noch der Bandenverlauf des vierwertigen Kations zu beobachten ist, als Ausgangspunkt, so vergrößert sich bei weiterer Zugabe von $\mathrm{CF}_{3} \mathrm{COOH}$ die Aufspaltung der beiden Teilbanden. Bei einer Konzentration von etwa $15 \% \mathrm{CF}_{3} \mathrm{COOH}$ erhöht sich die Differenz um etwa $1.6 \mathrm{~nm}$. Dabei verändert sich aber die Bandenform deutlich, wie es in Abb. 11 a dargestellt ist und 

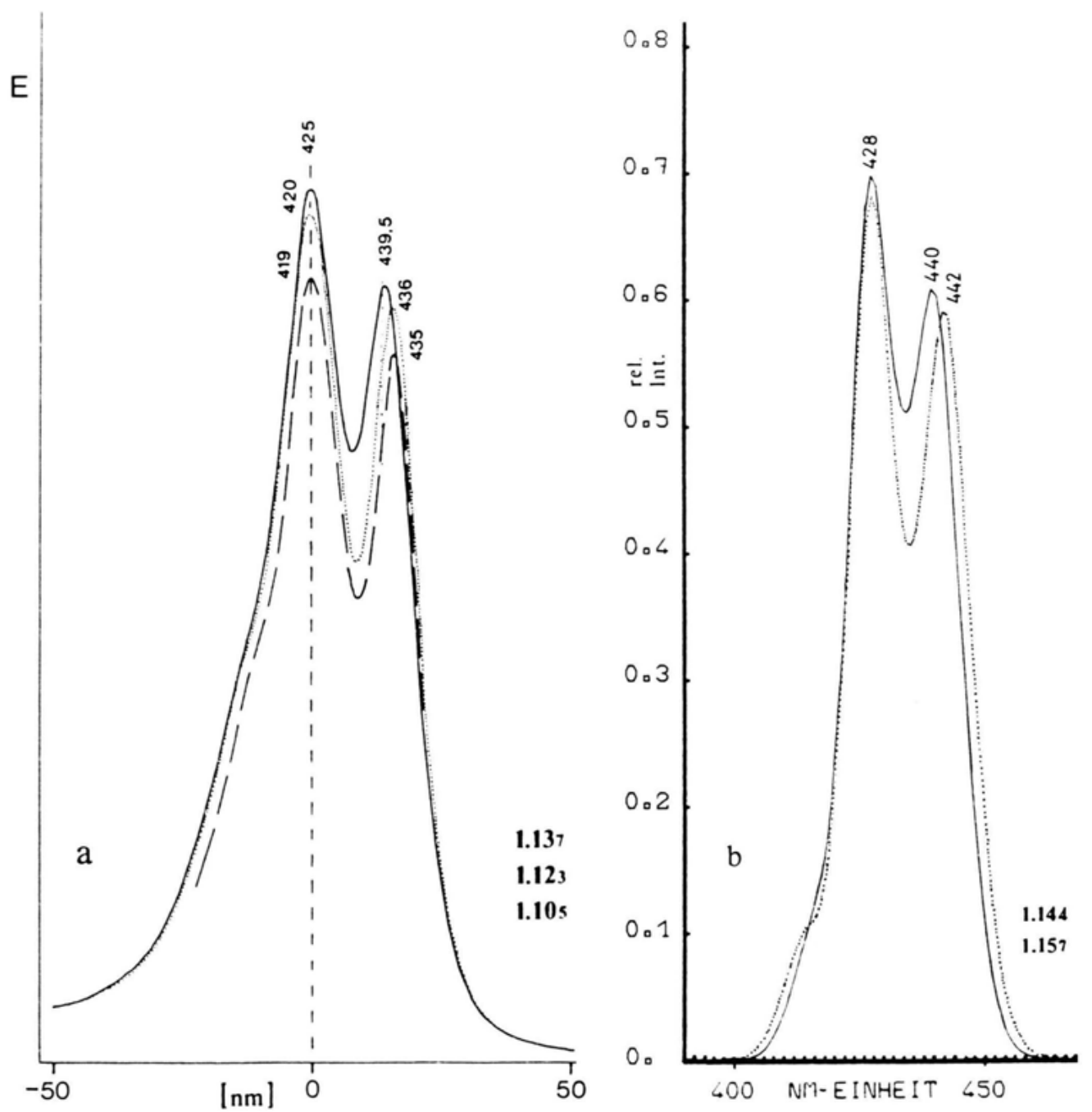

Abb. 11. Abhängigkeit der Aufspaltung der Soret-Bande vom Lösungsmittel. a: $\mathbf{2}$ a in $\mathrm{CHCl}_{3} / 1 \% \mathrm{TFA}(-), \mathrm{CHCl}_{3} /$ $\sim 8 \%$ TFA $(\cdots \cdots)$ und $\mathrm{CHCl}_{3} / \sim 25 \%$ TFA $(\cdots-\cdots)$; zur Verdeutlichung wurden die hypsochromen Teilbanden auf eine Position gesetzt (Maximum $\left.i_{\text {hypso }}=0\right)$ (senkrechte Zahlen: die beobachteten Bandenlagen); b: für 2 a $\left(\Phi_{1}=90^{\circ}\right.$, $\left.\Phi_{2}=180^{\circ}\right)$ mit $k=1,75(-)$ und $k=2(\cdots \cdots)$ berechnete Soret-Banden. Die Extinktionsverhältnisse $E_{\text {hypso }} / E_{\text {batho }}$ sind rechts neben den Kurven.

durch die Änderung des Extinktionsverhältnisses von $E_{\text {hypso }} / E_{\text {batho. }}=1,137$ nach 1,105 zum Ausdruck kommt. Obwohl diese Differenzveränderung zwischen den beiden Teilbanden der Soret-Banden nur gering ist, entspricht dies bei der berechneten Kurve einer Änderung des Korrekturfaktors von $k=1,75$ nach $k=2,0$, was einer Verkleinerung des $D$-Wertes von 2,5 auf 2,19 äquivalent ist. Im Gegensatz zur experimentellen Bande ergibt sich hier aber auf Grund der Vergrößerung des Abstandes der berechneten Teilbanden eine Zunahme des Extinktionsver- hältnisses von $E_{\text {hypso }} / E_{\text {batho. }}=1,145$ auf 1,157 . Dieser Widerspruch sollte nur mit einer Konformationsänderung erklärlich sein. Betrachtet man die Stellung der Dipole $I$ und 3 in Abb. 7, so ergibt eine Vergrößerung des Winkels zwischen diesen eine Intensivierung der bathochromen Teilbande. Dies stände im Einklang mit einer stärkeren Abstoßung der positiven Ladungen und damit der beiden Porphinebenen. Ein derartiger Einfluß der „Ionenatmosphäre" des vierwertigen Kations wird weiter unten noch ausführlicher diskutiert. 


\section{Dimere mit der Struktur 3}

Da das konformative Verhalten der über eine Methylenbrücke $\beta$ - $\beta^{\prime}$-verknüpften Porphindimere der Struktur 3 nicht mehr so eindeutig zu klären ist, wie bei den vorherigen meso- $\beta$-verknüpften der Struktur 2, seien einige mögliche, signifikant unterschiedliche Konformationen mit den dazugehörigen Dipol-Wechselwirkungen vorgestellt. Mit dem StuartBriegleb-Kalottenmodell als Grundlage, wurden nur die Konformationen in die nähere Betrachtung einbezogen, bei denen keine sterische Behinderung eintrat, bzw. die sich nahestehenden Gruppen immer noch einen größtmöglichen Abstand aufweisen. Die Porphinringe wurden vorerst als vollständig planar angenommen, d.h. die Pyrroleinheiten sind trotz der sich sterisch behindernden vier Wasserstoffatome im Zentrum nicht verdrillt, worauf weiter unten aber nochmals eingegangen werden muß. In Abb. 13 und 14 sind einige berechnete SoretBanden für verschiedene Torsionswinkel $\Phi_{1}$ und $\Phi_{2}$ dargestellt und nachfolgend diskutiert.

So würde bei einer verhältnismäßig ,flachen“ Anordnung der beiden Ringe zueinander $\left(\Phi_{1}=\Phi_{2}\right.$ $\left.= \pm 20^{\circ}\right)($ Abb. 13a) - unter Berücksichtigung, da $\beta$ der Korrekturfaktor $k=1,75$ verwendet wird - die 2-4 Kopplung für die ,in-Phase"-Anordnung eine um $\sim 3,0 \mathrm{~nm}$ hypsochrom verschobene (bei $\sim 425$ $\mathrm{nm}$ ) intensive und für die ,gegen-Phase“-Anordnung eine um $\sim 3,0 \mathrm{~nm}$ bathochrom verschobene (bei $431 \mathrm{~nm}$ ) sehr schwache Teilbande ergeben. Die
Kopplung der Dipole 1 und 3 ergibt hingegen nur für die ,in-Phase"-Anordnung eine um $8 \mathrm{~nm}$ bathochrom verschobene intensive Bande bei $436 \mathrm{~nm}$. Eine derartige Soret-Bande wird für das vierwertige Kation von $\mathbf{3}$ a nicht beobachtet. Auch bei den Torsionswinkeln $\Phi_{1}=\Phi_{2}= \pm 40^{\circ}$ erhält man eine Kurvenform (Abb. 13b), die mit der beobachteten Soret-Bande nicht im Einklang steht. Führt man an beiden Bindungen der Methylenbrücke eine weitergehende konrotatorische Bewegung aus $\left(\Phi_{1}=\Phi_{2}=\right.$ $\pm 70^{\circ}$ ), so stehen die H-Atome der Methylenbrücke zu denen der benachbarten meso-Methylgruppe auf Lücke (bei geringfügig weitergehender Drehung $\left(\Phi_{1}=\Phi_{2}= \pm 80^{\circ}\right)$ beginnen sich die H-Atome dieser 20- und 20'-meso-Methylgruppen und $\operatorname{der} \beta$-HAtome in 3- und 3'-Position zu berühren). Die dazugehörigen Bandenlagen und relativen Intensitäten berechnen sich für die Kopplung der Dipole 1-3 für das vierwertige Kation von 3 a nun mit $439,4 \mathrm{~nm}$ und $417,2 \mathrm{~nm}$ und für die 2-4 Kopplung mit $425,8 \mathrm{~nm}$ und 430,2 nm. Dies ergibt eine Superposition wie sie in Abb. 13c dargestellt ist. Zwar zeigt sich schon eine große Änhlichkeit mit den SoretBanden der dimeren vierwertigen Kationen der Struktur 3, allerdings weicht das Extinktionsverhältnis der beiden Teilbanden von dem experimentellen ab. Außerdem würde diese Konformation eine starke sterische Behinderung für den Substituenten $\mathrm{R}$ an der Methylenbrücke mit den benachbarten Methylgruppen in 20- und 20'-Position ergeben. Diese drei Konformationen (Abb. 13a-c) würden auch mit

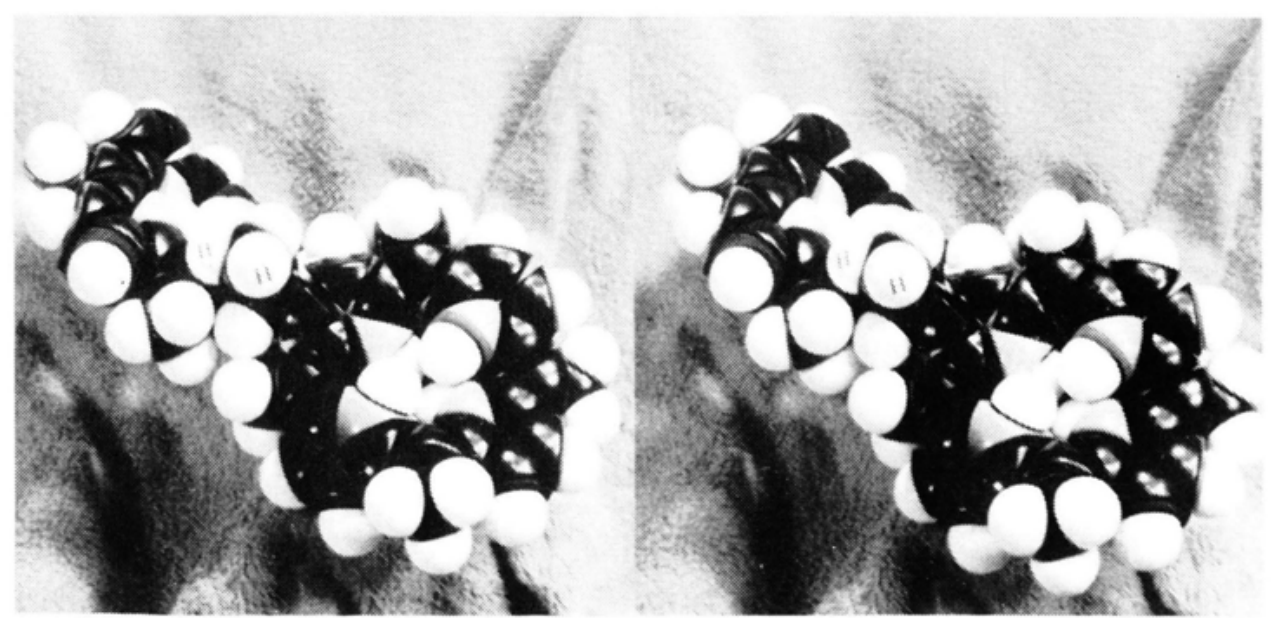

Abb. 12. Stereofoto, das den sterischen Einfluß der Methylgruppe an der Methylenbrücke auf die Konformationen in $\mathbf{3}$ d, $\mathbf{3}$ e und $\mathbf{3} \mathbf{f}$ widerspiegeln soll. 

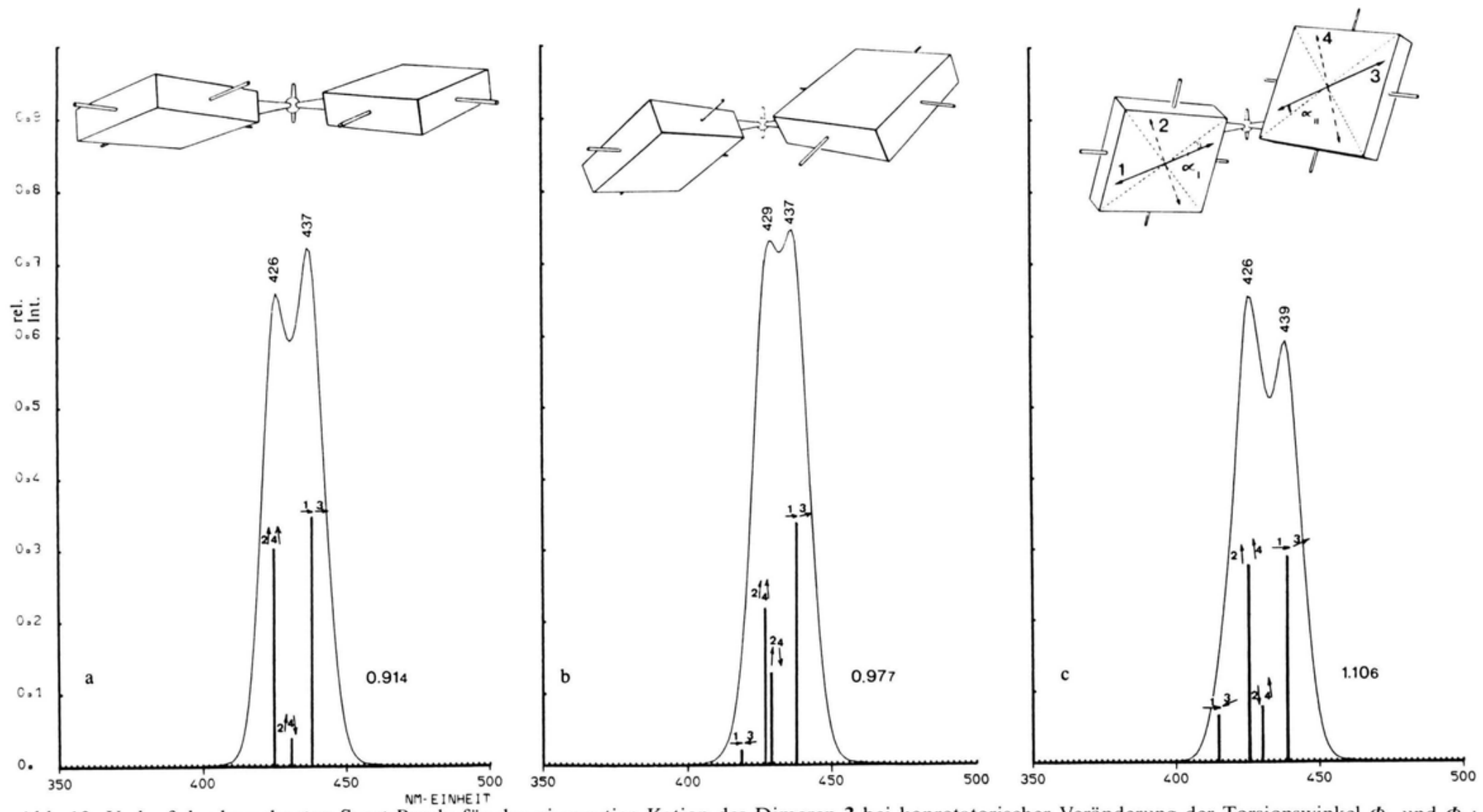

Abb. 13. Verlauf der berechneten Soret-Bande für das vierwertige Kation des Dimeren 3 bei konrotatorischer Veränderung der Torsionswinkel $\Phi_{1}$ und $\Phi_{2}$ eingeblendet die dazugehörige schematisierte Stellung der Porphinebenen mit den Dipolen 1, 2, 3 und 4. a: $\Phi_{1}=\Phi_{2}= \pm 20^{\circ}, \alpha_{1}=\alpha_{I I}=0^{\circ}:$ b: $\Phi_{1}=\Phi_{2}=$ $\pm 40^{\circ}, \alpha_{1}=\alpha_{I I}=5^{\circ} ; \mathrm{c}: \Phi_{1}=\Phi_{2}= \pm 70^{\circ}, \alpha_{I}=\alpha_{I I}=10^{\circ}$. Daneben das relative Extinktionsverhältnis $E_{\text {hypso }} / E_{\text {batho }} ; \lambda_{\text {mono }}=428 \mathrm{~nm} ; k=1,75$. 


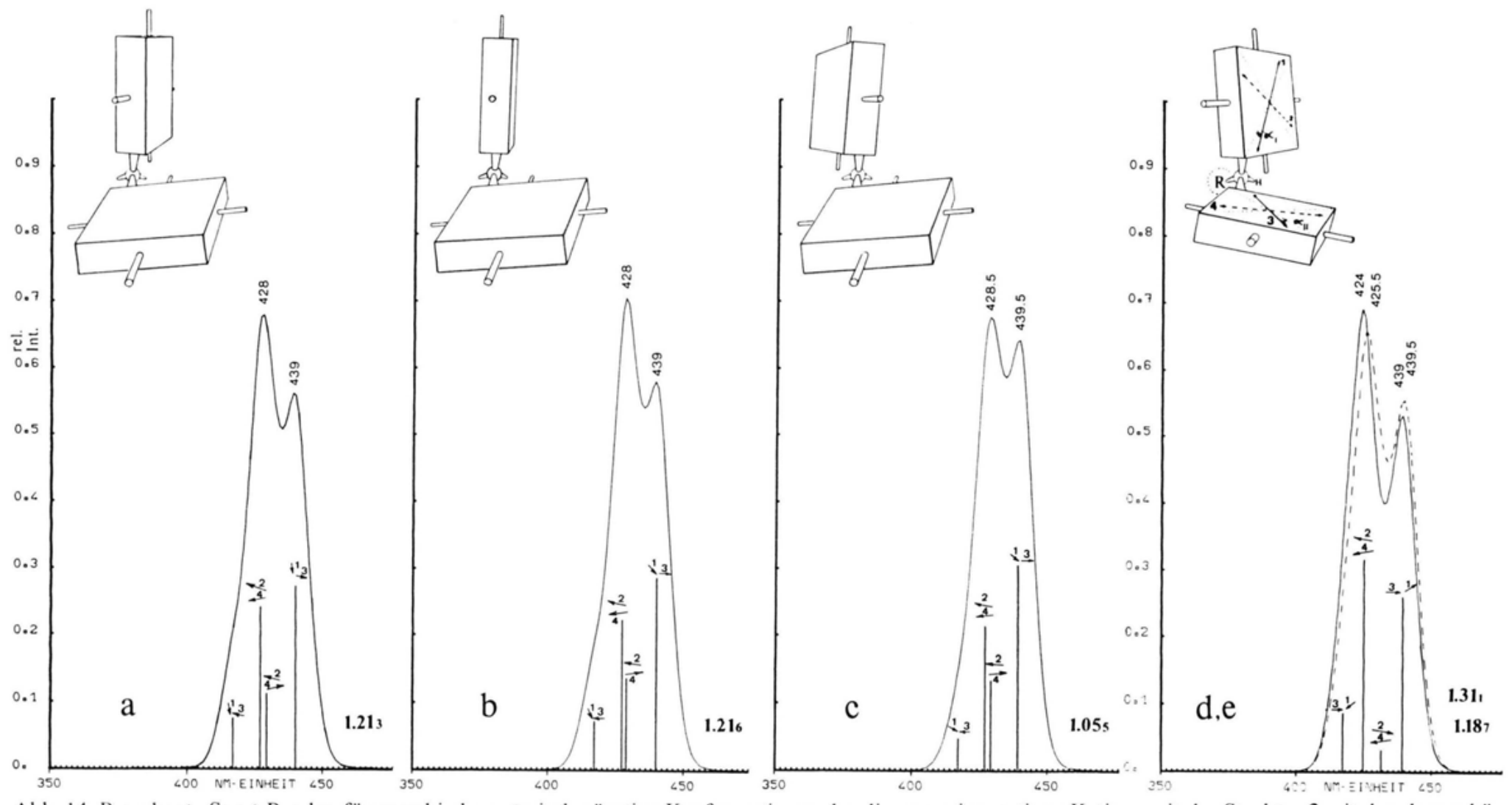

Abb. 14. Berechnete Soret-Banden für verschiedene sterisch günstige Konformationen des dimeren vierwertigen Kations mit der Struktur 3 mit den dazugehö-

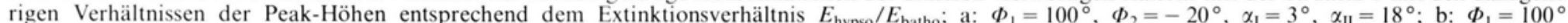
$\Phi_{2}=-5^{\circ}, \alpha_{1}=5^{\circ}, \alpha_{11}=16^{\circ} ;$ c: $\Phi_{1}=100^{\circ}, \Phi_{2}=20^{\circ}, \alpha_{1}=5^{\circ}, \alpha_{I I}=18^{\circ}$; d: $\Phi_{1}=70^{\circ}, \Phi_{2}=-30^{\circ}, \alpha_{1}=7^{\circ}, \alpha_{I I}=10^{\circ}$, mit ber. Lagen und f; e: $\Phi_{1}=75^{\circ}$, $\Phi_{2}=-22^{\circ}, \alpha_{1}=8^{\circ}, \alpha_{11}=12^{\circ}(\cdots) .\left(\lambda_{\text {mono }}=428 \mathrm{~nm} ; \dot{k}=1,75\right.$. 

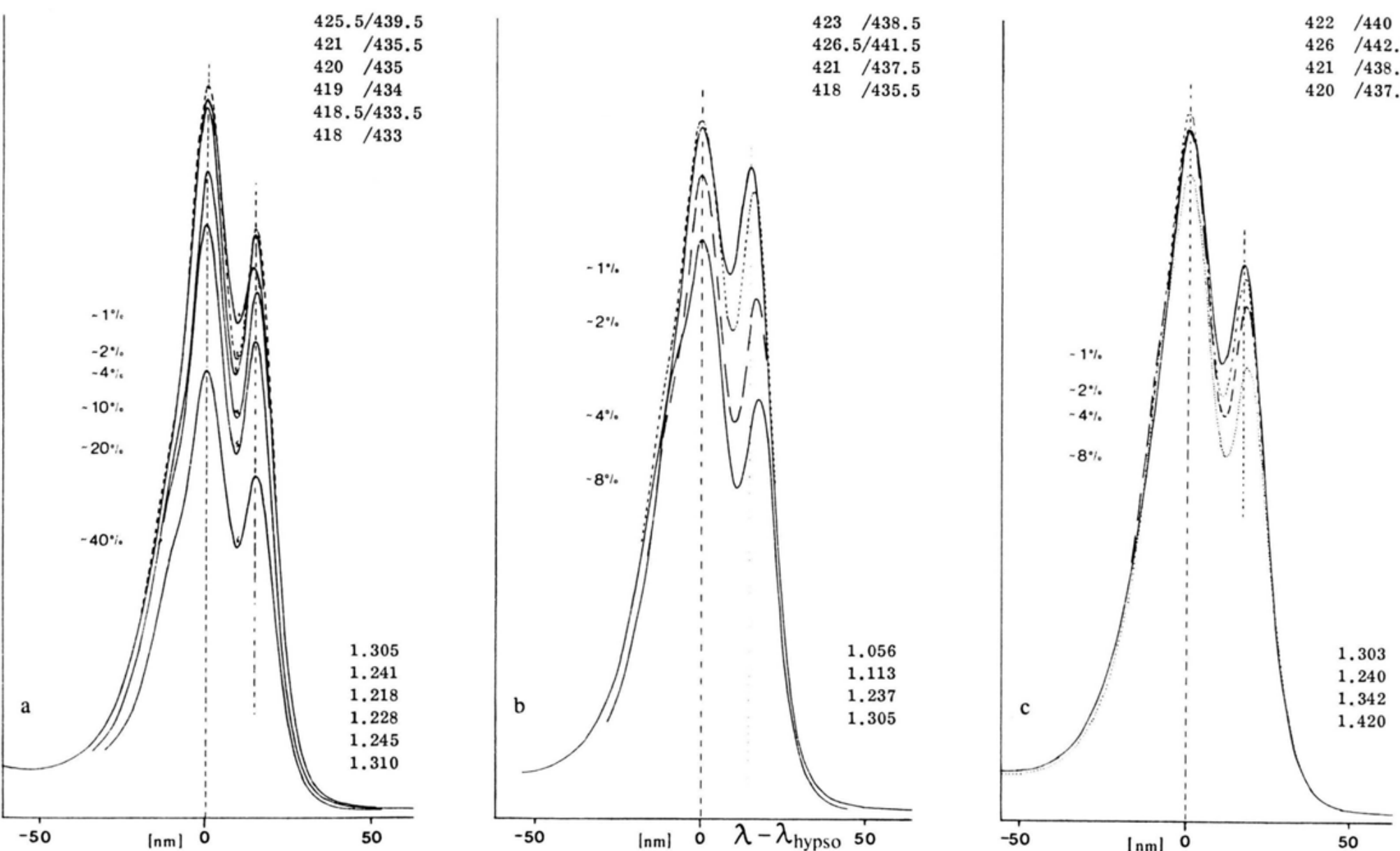

Abb. 15. Experimentell beobachtete Form der Soret-Banden der vierwertigen Kationen der Diporphinylmethanderivate 3a (a), 3d (b) und 3 $\mathrm{g}$ (c) in $\mathrm{CHCl}_{\text {, }}$ mit steigender Konzentration an $\mathrm{CF}_{3} \mathrm{COOH}$ (Prozentangabe neben der Bande). Die intensiveren hypsochromen Teilbanden wurden zum Vergleich alle auf die gleiche Position verschoben, um den Einfluß der steigenden Konzentrationen an TFA auszugleichen, der eine hypsochrome Verschiebung bewirkt. Zahlen oben rechts: beobachtete Lagen der hyposochr./bathochromen Teilbanden; unten rechts: rel. Verhältnis $E_{\text {hypso }} / E_{\text {batho }}$. 
Porphinringen, in denen die Pyrroleinheiten verdrillt sind, keine wesentlich sterisch günstigeren Verhältnisse an der Methylenbrücke ergeben.

Folgende unsymmetrische Konformationen erscheinen dagegen sehr günstig, da in ihnen die meso-Methylgruppen und die benachbarten Methylen-Brücken $\mathrm{H}$-Atome mit geringer sterischer $\mathrm{Be}$ hinderung wie dargestellt zueinander stehen. Für diese Konformationen errechneten sich die Bandenlagen und Intensitäten, deren dazugehörige Kurvensuperposition in Abb. 14 dargestellt sind.

Diese zeigen bis auf eine $\left(\Phi_{1}=100^{\circ} ; \Phi_{2}=20^{\circ}\right.$; Abb. $14 \mathrm{c}$ ) eine auffallende gute Übereinstimmung mit den experimentell beobachteten Soret-Banden der vierwertigen Kationen von $\mathbf{3} \mathbf{a}, \mathbf{3 d}$, und $\mathbf{3} \mathbf{g}$ (vgl. Abb. 15) in den Lösungsmitteln $\mathrm{CHCl}_{3}$ mit geringster Konzentrotion an $\mathrm{CF}_{3} \mathrm{COOH}$ und $\mathrm{CHCl}_{3} / \mathrm{konz}$ $\mathrm{H}_{2} \mathrm{SO}_{4}$. Danach scheint für 3a eine Konformation ähnlich der von $\left(\Phi_{1}=100^{\circ}, \Phi_{2}=-5^{\circ}\right)$ im Einklang mit den Betrachtungen über die sterisch günstigste Anordnung der Porphinringe und gleichzeitig der Wechselwirkung der ausgedehnten Dipole bzw. der daraus errechneten Soret-Bande zu sein, während für das Methylderivat $\mathbf{3 d}$ und das Phenylderivat $3 \mathrm{~g}$ die Konformation $\left(\Phi_{1}=100^{\circ}, \Phi_{2}=-20^{\circ}\right)$ und besonders $\left(\Phi_{1}=75^{\circ}, \Phi_{2}=-22^{\circ}\right)$ bzw. $\left(\Phi_{1}=\right.$ $70^{\circ}, \Phi_{2}=-30^{\circ}$ ) vorzuliegen scheinen. Auch stimmt die Beobachtung, daß sowohl in der berechneten wie auch experimentell beobachteten Soret-Bande des vierwertigen Kations von $\mathbf{3}$ a das Intensitätsverhältnis deutlich eine etwas intensivere hypsochrome Teilbande zeigt als bei denen der Dimeren $\mathbf{3 d}$ und $3 \mathbf{g}$ und die Aufspaltung beider Teilbanden deutlich geringer ist. Hinzu kommt die Tatsache, daß die Konformationen $\Phi_{1}=75^{\circ}, \Phi_{2}=-22^{\circ}$ bzw. $\Phi_{1}=$ $70^{\circ}, \Phi_{2}=-30^{\circ}$ besonders für größere Substituenten an der Methylenbrücke eine verhältnismäßig sterisch unbehinderte Situation ergeben.

In den Dimeren $\mathbf{3} \mathbf{d}, \mathbf{3} \mathbf{e}$ und $\mathbf{3} \mathbf{f}$ führt die Methylgruppe $\left(\mathrm{R}=-\mathrm{CH}_{3}\right)$ zu einer starken Einschränkung der konformativen Möglichkeiten im Vergleich zu 3a (Abbildung 12). Stellt man am Kalottenmodell die sich nahestehenden H-Atome der mesoMethylgruppen der 20-, 20'-Positionen, sowie der Methylgruppe an der Brücke ,auf Lücke“ zum Wasserstoffatom der Methylenbrücke und zu dem der benachbarten $\beta$-Position, so entspricht dies einer sterisch günstigen Konformation mit den Torsionswinkel $\Phi_{1}=75^{\circ}$ und $\Phi_{2}=-22^{\circ}$, wie sie in Abb. $14 \mathrm{e}$ dargestellt ist.
Einen zur Methylgruppe sehr ähnlichen Einfluß auf das konformative Verhalten der Dimeren mit der Struktur 3 hat ein Phenylrest. Dies findet seinen Ausdruck in dem sehr ähnlichen spektroskopischen Verhalten der beiden Dimeren 3d und 3g. Die Konformation, in der der Phenylrest und die beiden Porphinringe die geringste sterische Behinderung aufweisen, weicht nur geringfügig von der des Methylderivates 3d ab. Die dazugehörigen Torsionswinkel betragen $\Phi_{1} \approx 70^{\circ}, \Phi_{2} \approx-30^{\circ}$ bzw. $\Phi_{1} \approx-30^{\circ}$ und $\Phi_{2} \approx 70^{\circ}$.

Obwohl diese Änderung der Konformation im Verhältnis zur vorhergehenden von $\mathbf{3} \mathbf{d}-\mathbf{3} \mathbf{e}\left(\Phi=75^{\circ}\right.$, $\Phi_{2}=-22^{\circ}$ bzw. $\Phi_{1}=-22^{\circ}, \Phi_{2}=75^{\circ}$ ) nur sehr gering erscheint, zeigt die dazugehörige berechnete Kurve einen deutlichen Unterschied (vgl. Abb. 14d) im Intensitätsverhältnis von hypsochromer zu bathochromer Teilbande. Die Übereinstimmung mit der beobachteten Soret-Bande ist sehr gut (vgl. Abbildung $15 \mathrm{c}$ ). In dieser speziellen Konformation hat eine geringe Verkleinerung des Winkels zwischen den beiden Porphinbindungen an der Methylenbrücke, die durch die Phenylgruppe hervorgerufen werden kann, eine Verkleinerung des Winkels zwischen den Dipolen 1 und 3 zu Folge, wodurch die Intensität der bathochromen Teilbande zugunsten der hypsochromen verringert wird. Hinzukommt, da $\beta$ in dieser Konformation auch eine geringfügig stärkere Kopplung zwischen den Dipolen 2 und 4 eintritt, deren hypsochrome Verschiebung der intensiven Teilbande der ,In-Phase“-Anordnung noch etwas stärker als bei der Konformation des Methylenderivates $\mathbf{3} \mathbf{d}$ ist und somit die Differenz der beiden Teilbanden geringfügig vergrößert wird. Dies steht im Einklang mit den experimentell beobachteten Unterschieden der Soret-Banden von 3d und $\mathbf{3 g}$ (Abbildung $15 \mathrm{~b}$ und $\mathrm{c}$ ).

Wird die Methylgruppe durch den Phenylring ersetzt, so beobachtet man eine deutliche bathochrome Verschiebung der aufgespaltenen SoretBande. Vergleicht man den Einfluß der $\beta$-Substituenten $\mathrm{R}^{1}$ der Monomeren $\mathbf{1} \mathbf{a}-\mathbf{1} \mathbf{h}$, so scheint es gerechtfertigt, dieser Phenylgruppe in den Dimeren $\mathbf{3 g}$ und $\mathbf{3 h}$ eine zusätzliche bathochrome Verschiebung von etwa $3,0 \mathrm{~nm}$ zuzuschreiben.

Eine völlig andere Situation scheint für das Dimere $\mathbf{3} \mathbf{g}$ in dem Lösungsmittelgemisch $\mathrm{CHCl}_{3}$ /Ethanol $(1: 1)$ vorzuliegen, in dem die Intensität der bathochromen Teilbande deutlich verkleinert ist (Abbildung 16). Dabei ist die Lage der Banden 


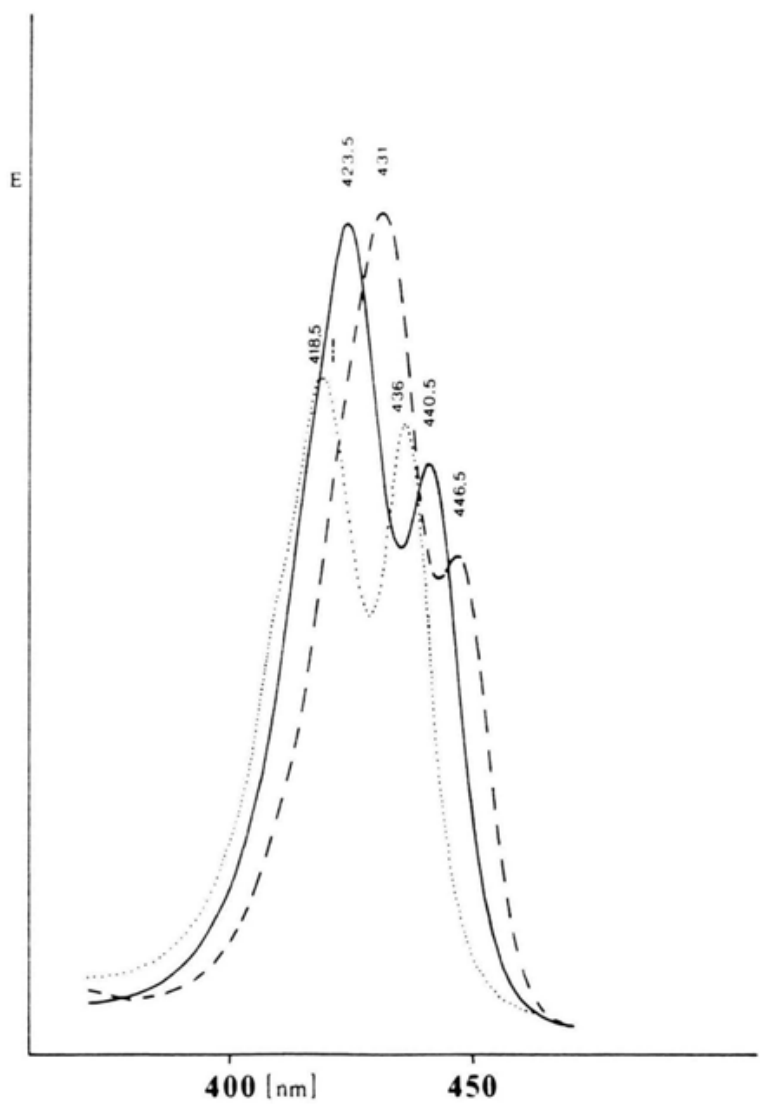

Abb. 16. Die Soret-Banden des vierwertigen Kations des phenylsubstituierten Dimeren $\mathbf{3} \mathbf{g}$ in verschiedenen Lösungsmitteln: - in $\mathrm{CHCl}_{3} /$ Ethanol $(1: 1)+1 \% \quad 48 \%$ $\mathrm{H}_{2} \mathrm{SO}_{4} ;---$ in $\mathrm{CHCl}_{3} /$ Ethanol $(1: 1)+1 \%$ konz. $\mathrm{HCl}$; $\cdots$ in konz. $\mathrm{H}_{2} \mathrm{SO}_{4}$.

noch von der Art der Anionen $\left(\mathrm{Cl}^{-}\right.$bzw. $\left.\mathrm{HSO}_{4}^{-}\right)$ bzw. der hinzugefügten Säure (konz. $\mathrm{HCl}$ und $\left.48 \% \mathrm{H}_{2} \mathrm{SO}_{4}\right)$ abhängig. Aber auch in Chloroformlösung bei steigender Konzentration an Trifluoressigsäure (TFA) wird für $\mathbf{3} \mathbf{g}$, dem Methylderivat $\mathbf{3 d}$ sowie 3a ein sehr ähnliches Verhalten beobachtet: mit steigender Konzentration an TFA nimmt die Intensität der bathochromen Teilbande $a b$ und gleichzeitig die der Schulter an der hypsochromen Teilbande deutlich zu, während für $\mathbf{2}$ a ein entgegengesetztes Verhalten beobachtet wurde. Geht man davon aus, daß dieses Modell der Wechselwirkung ausgedehnter Dipole in dieser einfachen Form anwendbar ist - wofür weiter unten noch andere Beispiele dimerer Porphine den Beweis liefern dann kann die Veränderung der Soret-Banden dieser Dimeren nur als Folge einer Konformations- änderung der beiden Porphinringe zueinander gedeutet werden: Da es sich bei den hier beschriebenen Dimeren um die Verknüpfung zweier Dikationen handelt, wird die Situation der „Ionenatmosphäre" [46] einen Einfluß auf die Konformation haben, bedenkt man den geringen intramolekularen Abstand zwischen den beiden zweifach-positiv geladenen Zentren von ca. 9,5-10 А. So wird in diesem Fall der Grad der Dissoziation der hier vorliegenden Ionenpaare und die Art deren Solvatation (damit verbunden deren Radius) eine wesentliche sterische Wirkung auf die Stellung der beiden Porphinringe ausüben. Da dies von der Größe der Dielektrizitätskonstanten des Lösungsmittels, von den Dipolstärken einzelner Gruppen und von der Möglichkeit der Bildung von Wasserstoffbrücken abhängig ist, wird somit auch die Kopplung der Übergangsmomente hiervon beeinflußt.

So ist bekannt, daß in Lösungsmitteln mit kleiner Dielektrizitätskonstante nach der Bjerrumschen Theorie eine starke Assoziation der Ionenpaare vorliegt, d.h. in Lösungsmitteln wie Chloroform ( $\mu=1,01$ Debye; Dielektrizitätskonstante $\varepsilon=4.81$ ) oder Ethanol $(\varepsilon=24.3 ; \mu=1.69$ Debye $)$ sollte sich das Anion in sehr geringer Distanz zum zweifachpositiv-geladenen Zentrum des Porphinringes aufhalten, wobei auch der Grad der Solvation der Ionen mit kleiner werdender Dielektrizitätskonstante abnimmt [46]. Dies zeigt sich durch eine sehr deutliche Differenz der Lage und einem etwas veränderten Intensitätsverhältnis der Soret-Teilbanden

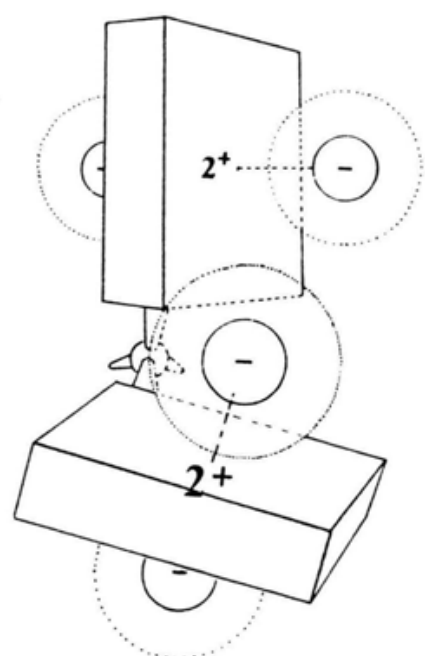

Abb. 17. Schematisierte Anordnung der Anionen am vierwertigen Kation der Struktur 3. 
und auch der Q-Banden in Chloroform/Ethanol (1:1)-Lösung in Abhängigkeit der beiden Anionenarten $\mathrm{Cl}^{\ominus}$ bzw. $\mathrm{HSO}_{4}^{\ominus}$. (Da mit $48 \% \mathrm{H}_{2} \mathrm{SO}_{4}$ angesäuert wurde, sollte es sich hierbei um das Hydrogensulfat-anion handeln.)

So liegt die Vermutung nahe, daß beim $\left[(\mathrm{TMP}) \mathrm{H}_{4}\right]^{2+} \cdot 2 \mathrm{Cl}^{\ominus}$ in Chloroformlösung (vgl. Tab. 1), das Chlorid-Ion ohne Solvathülle direkt an die $\mathrm{N}-\mathrm{H}$-Gruppen der Pyrroleinheiten assoziiert ist. Daraus sollte eine starke Neigung der Ebenen dieser zur Durchschnittsebene des gesamten Porphinringes folgen, wie sie im kristallinen Zustand des (TPP) $\mathrm{H}_{4}^{2+}$ beobachtet wurde [50]. Obwohl hiermit eine Störung des konjugierten Elektronensystems verbunden sein sollte, wird gleichzeitig eine stabilisierende Wirkung auf den angeregten Zustand ausgeübt, da die Abstoßung der beiden positiven Ladungen im Innern des Ringes verringert wird. Im angeregten Elektronenzustand $\mathrm{e}_{\mathrm{g}}$ befinden sich an den N-Atomen Anhäufungsstellen der Elektronenwolken [35, 47]. Diese werden durch Protonierung stabilisiert [48] und dadurch die Anregungsenergie der Soret-Bande $\left(\mathrm{a}_{1}-\mathrm{e}_{\mathrm{g}}\right.$-Übergang) langwellig verschoben. Ebenso sollten polare, wasserstoffbrückenbildende Lösungsmittel auf diesen polaren Anregungszustand wirken, was auch beobachtet wird (vgl. Tabelle 1). Dies wird auch durch das erheblich bathochrom verschobene Spektrum des sterisch stark bedrängten meso-Tetraphenyl-octaethylporphins bestätigt, das bereits mit Wasser zum Dikation reagiert [49].

Weiterhin ist zu berücksichtigen, daß sich bei der Protonierung der freien Base die vier Wasserstoffatome im Zentrum des Porphinringes auf Grund ihres van-der-Waals-Radius sterisch behindern. Als Folge kann ein mehr oder weniger starkes Herausdrehen der Pyrroleinheiten aus der Ebene des Porphinringes erfolgen, wie es in einer Kristallstruktur des (TPP) $\mathrm{H}_{4}^{2+}[50]$ beobachtet wurde. Wohingegen in einem Dikation des (OEP) $\mathrm{H}_{4}^{2+}$ die Abweichung der Pyrrolebenen aus der Ebene des Porphinringes
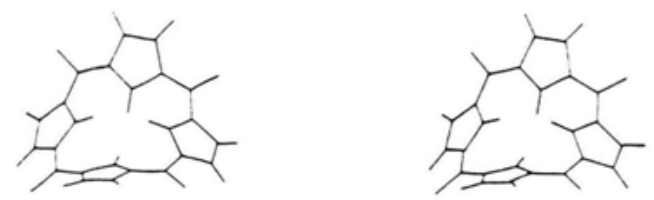

Abb. 18. Stereobild der Abweichung der Pyrrolebenen aus der Ebene des Porphinringes, wie es in der Kristallstruktur des (TPP) $\mathrm{H}_{4}^{2+}$ gefunden wird [50]. nur sehr gering ist [51]. Für ein dem Dikation des TPP vergleichbares sterisches Verhalten der Pyrrolringe im Dikation des TMP 1 a und seiner Derivate spricht die große Ähnlichkeit dieser Elektronenspektren. Während in den Spektren der Dikationen $\beta$-substituierter Porphyrine die kurzwelligere der beiden Q-Banden die intensivere ist, ist es bei den meso-aryl und meso-alkyl-substituierten Porphinen umgekehrt (Bande I ist intensiver als Bande II). Dieser als ms-Spektraltyp bezeichnete Bandenverlauf wird als Folge der verhältnismäßig großen Neigung der Ebenen der Pyrroleinheiten zu der der Porphinebene gedeutet [50]. Ebenso wird die bathochrome Verschiebung der Soret-Bande der Dikationen der meso-Arylporphine von $\sim 20-25 \mathrm{~nm}$ gegenüber ihrer freien Basen gedeutet, während bei den $\beta$-substituierten Porphinen diese Differenz nur $\sim 5 \mathrm{~nm}$ beträgt. Die Soret-Bande des Dikations von 1 a wird bei gleichen Aufnahmebedingungen wie beim (TPP) $\mathrm{H}_{4}^{2+}$ immerhin noch $15 \mathrm{~nm}$ bathochrom verschoben. Dies ist auch insofern beachtlich, da die beobachtete bathochrome Verschiebung von $26 \mathrm{~nm}$ des (TPP) $\mathrm{H}_{4}^{2+}$ mit einer verstärkten Mesomerie des Phenylrestes mit dem Porphinkern erklärt wird, die durch die Verdrillung der Pyrroleinheiten begünstigt wird [50]. Ein vergleichbarer MesomerieEffekt ist bei der ms-Methylgruppe nicht möglich. Deshalb sollte dies auf die Neigung der Pyrroleinheiten im Dikation zurück zu führen sein. Die unten erwähnten Konformationsenergieberechnungen zeigten nämlich eine auffallende sterische Wechselwirkung der ms-Methylgruppen mit den benachbarten $\beta$-ständigen Wasserstoffatomen im ebenen Porphinsystem von $\mathbf{1}$ a, wodurch eine solche Neigung bevorzugt wird.

So betrug die Energiedifferenz zwischen der energetisch ungünstigeren Konformation $\mathrm{A}$ und der Konformation B immerhin $2,9 \mathrm{kcal} / \mathrm{mol}$ für die einzelne Methylgruppe [42], wenn man die Bindungslängen und Winkel für das meso-Tetramethylporphin zu Grunde legte, wie sie in der Kristall-

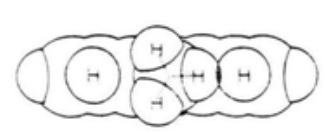

A

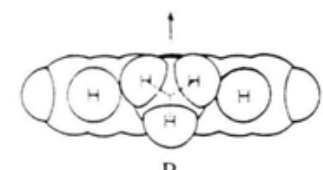

B
Schema 6 

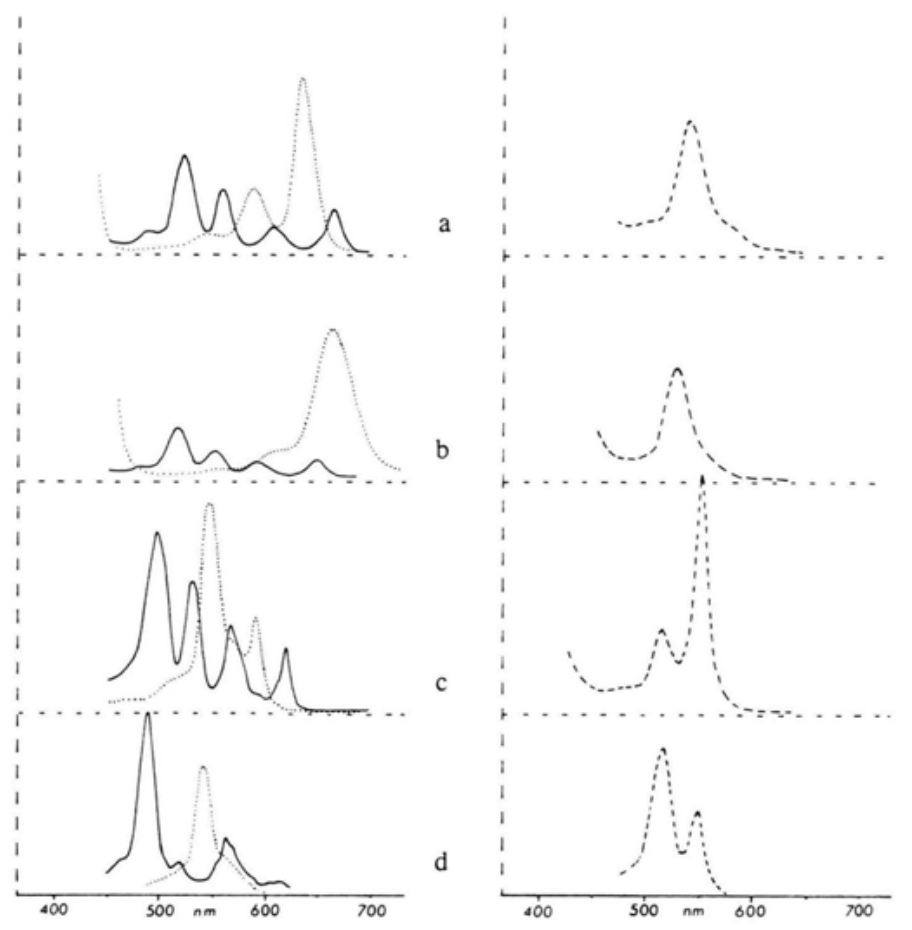

Abb. 19. Absorptionsspektren der freien Basen $(-)$, der Dikationen $(\cdots \cdots)$ und der Nickelkomplexe (---) des meso-Tetramethylporphins (a), des meso-Tetraphenylporphins (b), des Octaethylporphins (c) und des Porphins (d).

struktur des Ni-TMP 1 b gefunden werden [43]. Selbst wenn die Bindungslänge der meso-Methylgruppe zum meso-C-Atom von $1.518 \AA$ auf $1.568 \AA$ vergrößert wird, beträgt die Energiedifferenz immer noch $2,4 \mathrm{kcal} / \mathrm{mol}$. Auch wenn in der Kristallstruktur ein fast planarer Porphinring gefunden wird [43], begünstigt durch die Packung im Kristall, sollte in Lösung der meso-Substituent aus der Porphinebene herausgedrängt werden. Dieser Effekt sollte auf Grund der unsymmetrischen Anordnung der $\mathrm{H}$ Atome der Methylgruppe zu den benachbarten $\beta$-HAtomen des Porphinringes, wie in der Anordnung B deutlich erkennbar, eventuell sogar größer sein als bei dem analog symmetrisch senkrecht zur Ebene angeordneten Phenylring im meso-Tetraphenylporphin, wodurch sich auch der ausgeprägte Unterschied der UV-Spektren im sichtbaren Bereich erklären ließe, der als ms-Spektraltyp bezeichnet wird.

Diese Wechselwirkung wird auch durch den Vergleich der Bindungslängen und Winkel bestätigt, die in den Kristallstrukturen von Porphin, ms-TPP und meso-Tetra-n-propylporphin gefunden werden [52]. Danach wird das Brücken-C-Atom durch die mesoSubstituenten deutlich nach außen gezogen.
Aber auch ${ }^{1} \mathrm{H}-\mathrm{NMR}$-spektroskopische Untersuchungen über den Einfluß von $\beta$ - und meso-Substituenten auf die Verschiebung der N-H-Protonen verschiedener Porphine in Trifluoressigsäure bestätigen die hier diskutierte Verdrillung der Pyrroleinheiten in den Dikationen [53]. Während z. B. die $\mathrm{N}-\mathrm{H}$-Protonen des größten Teils aller $\beta$-substituierten Porphine wie z.B. Octamethyl-, Etioporphyrin um $\delta=-4,8 \mathrm{ppm}$ erscheinen, werden die für mesoTetramethylporphin bei $\delta=-3,01 \mathrm{ppm}$ und die für meso-Tetraphenylporphin bei $\delta=-2,07 \mathrm{ppm}$ in TFA beobachtet.

Dies bestätigt die auf Grund der Elektronenspektren gemachten Beobachtungen und zeigt, da $\beta$ die $\beta$-substituierten Porphine auch als Dikationen planarer als die meso-substituierten sind, was bei einigen Dimeren des Etioporphyrin weiter unten noch zu berücksichtigen sein wird.

Nimmt man deshalb eine leicht verdrillte Anordnung der Pyrroleinheiten in diesen Porphindikationen an, so sollte es in Lösung auch vier verschiedene Konformationen der Pyrroleinheiten geben, ähnlich denen, die für Porphyrinogene nachgewiesen wurden [54]: 


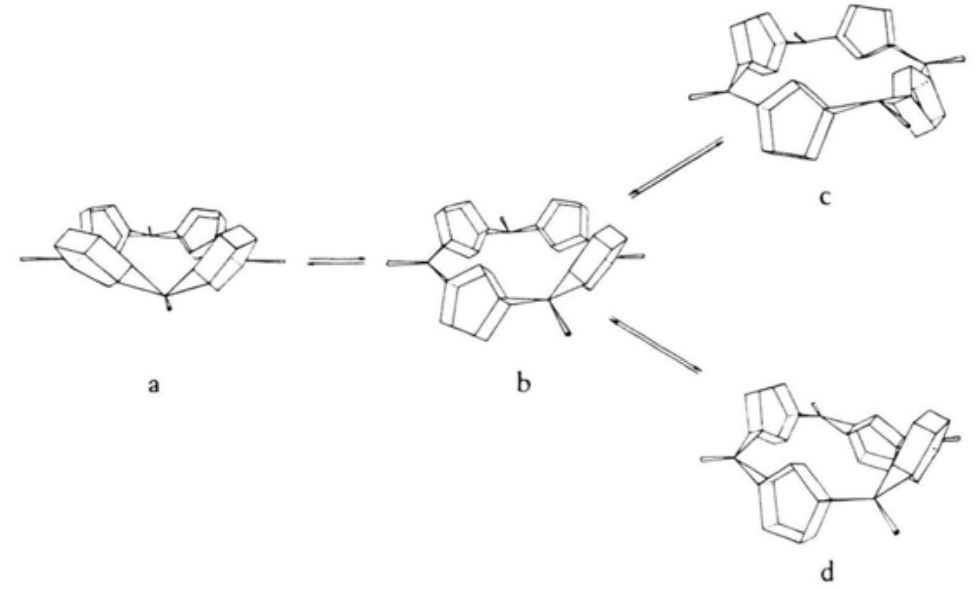

Abb. 20. Die vier grundlegenden schematisierten Stellungen der Pyrroleinheiten im Porphindikation, wenn diese nicht in der Porphinebene liegen (die Neigung der Pyrrolebenen ist zur Verdeutlichung übertrieben dargestellt, vgl. Abbildung 18).
Bei den Porphyrinogenen konnte gezeigt werden, $\mathrm{da} ß$ besonders die Liegestuhl-Konformation (Abb. $20 \mathrm{c})$ sehr stabile Wasserstoffbindungen zum Methanol bildet, in der beide gleichgerichtete benachbarte $\mathrm{N}-\mathrm{H}-\mathrm{Gruppen}$ zum gleichen Sauerstoffatom des Alkohols gebunden sind, während die Sattelkonformation analog Abb. $20 \mathrm{~d}$, nur schwache und instabile Wasserstoffbrückenbindungen zum Alkohol ausbildet. Auf Grund der konformativen Ähnlichkeit in der Stellung der Pyrroleinheiten sollten ähnliche Möglichkeiten für diese Dikationen diskutabel sein.

Hieraus ergeben sich für die Dimeren der Struktur 3 auf einfachem Weg unterschiedliche Konformationen, die auch nur eine geringe Energiedifferenz aufweisen sollten. Dies sei in den stark schematisierten Anordnungen A, B und C der Pyrrolebenen ( $\cdots$ ) gegenüber den Porphinebenen $\left(\sqsubset 2^{+} \sqsupset\right)$ dargestellt, bei denen die Torsionswinkel $\Phi_{1}$ und $\Phi_{2}$ unverändert bleiben. Die Porphinebene, die durch die vier meso-Positionen definiert wird, schließt dabei mit der Ebene des Pyrrolringes rein fiktiv einen Winkel von $15^{\circ}$ ein:

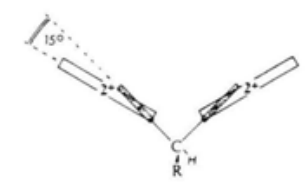

Schema 7 A

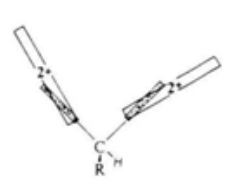

B

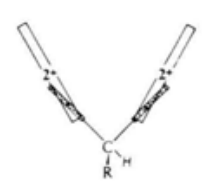

C
Behält man bei diesen drei prinzipiell unterschiedlichen Positionen A, B und C die für die planare Anordnung der Pyrroleinheiten gefundenen günstigsten Torsionswinkel $\Phi_{1}$ und $\Phi_{2}$ bei, wodurch sich die sterische Situation der Molekülgruppen direkt an der Methylenbrücke nicht wesentlich ändert, so ergeben sich z. B. die in Abb. 21 stereoskopisch dargestellten Konformationen A, B und C.

Die sich daraus ergebende unterschiedliche Stellung der Dipole zueinander könnte, bei einer rein qualitativen Abschätzung, die verschiedenen Formen der Soret-Banden der vierwertigen Kationen mit der Struktur $\mathbf{3}$ in den verschiedenen Lösungsmitteln erklären: Verwendet man reine konzentrierte $\mathrm{H}_{2} \mathrm{SO}_{4}$ als Lösungsmittel, die eine sehr große Dielektrizitätskonstante von 110 besitzt, so führt dies zu einer Ausdehnung der Ionenatmosphäre besonders der Anionen $\left(\mathrm{HSO}_{4}^{-}\right)$[55]. Daraus folgt, daß die effektive Gesamtladung der einzelnen Porphinringe steigt und sich die vier positiven Ladungen stärker abstoßen. Dies begünstigt, wie bei Polyelektrolyten beobachtet [56], eine mehr gestreckte Konformation wie in A: da die Dipole 1 und 3 nur leicht gewinkelt hintereinander liegen, ergibt dies eine intensive bathochrome Bande, während die Wechselwirkung der fast orthogonal zueinander stehenden Dipole 2 und 4 eine intensivere kaum verschobene Bande ergäbe (Bezeichnung der Dipole wie in Abbildung $14 \mathrm{~d}, \mathrm{e})$.

Eine ähnliche Situation sollte auch vorliegen, wenn die Chloroformlösung der Phenylsubstituierten freien Base $\mathbf{3 g}$ mit konzentrierter 95-97\% $\mathrm{H}_{2} \mathrm{SO}_{4}$ ausgeschüttelt wird. Erstaunlicherweise verbleibt das dabei entstandene vierwertige Kation in der Chloroformphase, was auf ein insgesamt unpolares Ionenpaar schließen läßt. Über die Art der Anionen können nur Vermutungen angestellt wer- 

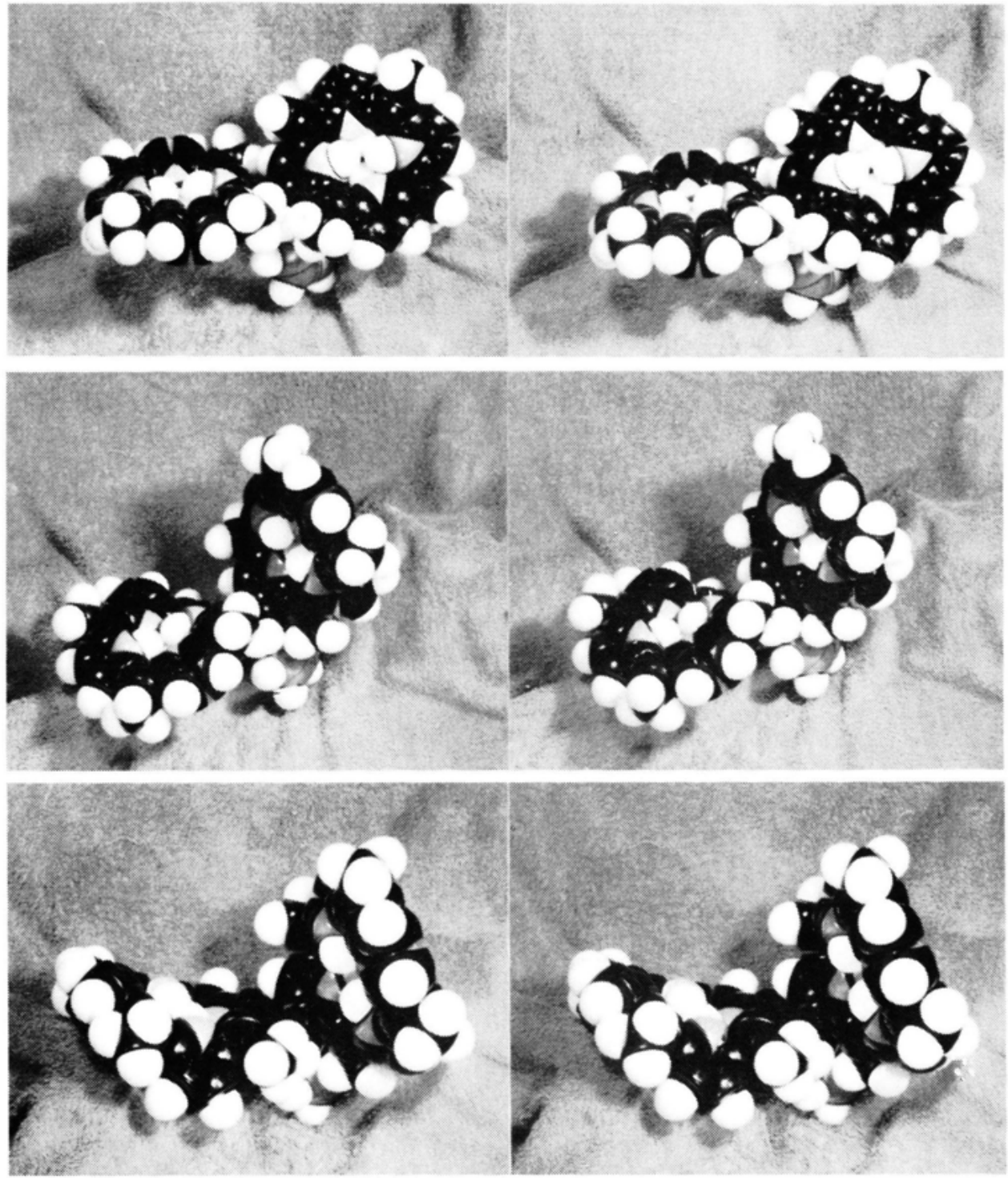

Abb. 21. Stereobilder der Konformationen A, B und C, die durch die möglichen Stellungen der Pyrrolebene zur Porphinebene hervorgerufen wird, wie oben schematisiert dargestellt ist. Die Torsionswinkel $\Phi_{1}$ und $\Phi_{2}$ an der Methylenbrücke sind stets gleich (Erklärung im Text).

den. So beobachtet man für Pyridiniumhydrogensulfat in erhöhter Konzentration von $\mathrm{H}_{2} \mathrm{SO}_{4}$ in Nitromethan die Bildung von $\mathrm{PyH}^{+} \ldots \mathrm{HSO}_{4}^{-}\left(\mathrm{H}_{2} \mathrm{SO}_{4}\right)_{2}$ Ionenpaaren [57]; ähnliches wäre auch in diesem Fall denkbar.

In $\mathrm{CHCl}_{3} / \sim 1 \% \mathrm{CF}_{3} \mathrm{COOH}$ sollte eine Konformation wie in B vorliegen: da die Dipole 1 und 3 nun gewinkelt zueinander sind, ergibt dies eine schwächere bathochrome Teilbande für die ,in-Phase"-
Anordnung und da die ,gegen-Phase"-Anordnung eine geringe Intensität liefert, ergibt dies eine schwache hypsochrome Bande. Diese träte als Schulter der intensiven hypsochromen Teilbande auf, die durch die parallele Anordnung der beiden Dipole 2 und 4 erzeugt wird.

Vorausgesetzt, daß auch für die Dikationen mit stärker verdrillten Pyrrolringen in erster Näherung immer noch die freie Drehbarkeit der beiden senk- 
recht zueinanderstehenden Dipole im monomeren Porphinring bzw. deren eingangs erläuterte Ausrichtung im Dimeren zulässig ist, dann ist der Verlauf der Soret-Banden in dem Lösungsmittelgemisch Chloroform-Ethanol $(1: 1)$ und auch eventuell Chloroform/Trifluoressigsäure (1:9) am besten durch eine Konformation ähnlich der in $\mathrm{C}$ zu erklären: Der Winkel zwischen den Dipolen 1 und 3 wäre im Bereich von etwa $90^{\circ}$, dies ergäbe eine hypsochrome und eine gleichstarke bathochrome Bande. Während für die Wechselwirkung der immer noch mehr parallel angeordneten Dipole 2 und 4 in erster Linie nur die in Phase-Anordnung eine intensive ebenfalls leicht hypsochrome verschobene Teilbande ergäbe, die mit der hypsochromen Bande der Wechselwirkung $1-3$ überlagern würde. Das Resultat für diese Soret-Bande wäre eine sehr intensive hypsochrome und eine schwächere bathochrome Teilbande mit einem Intensitätsverhältnis von etwa $(1+2): 1$, wie es auch beobachtet wird. Diese muschelförmige Konformation C könnte durch Wasserstoffbrückenbindungen mit Ethanol bzw. Trifluoressigsäure begünstigt werden.

Aber auch bei einer planaren Anordnung der Porphinebenen im vierwertigen Kation, wie sie für die Berechnung als Grundlage verwendet wurde (vgl. Abb. 19d, e), würde eine stärkere Abstoßung der beiden zweifach-positiv geladenen Zentren zu einer Aufweitung des Bindungswinkels an der Methylenbrücke führen und damit verbunden zu einer Vergrößerung des Winkels zwischen den Dipolen 1 und 3, was zu einer Intensivierung der bathochromen Teilbande in $\mathrm{H}_{2} \mathrm{SO}_{4}$ führt.

Auch für das Dimere 3 a wurde eine Konformations-Energie-Berechnung durchgeführt [42], um die hier gemachten Interpretationen weiter zu überprüfen. Auf der Grundlage der Bindungslängen und Winkel, wie sie schon für die Porphinringe des Dimeren der Struktur 2 verwendet wurden, wurde die Konformationsenergie als Funktion der Bindungswinkel $\Phi_{1}$ und $\Phi_{2}$ berechnet. Die beiden zur Methylenbrücke direkt benachbarten meso-Methylgruppen wurden dabei in ihrer Stellung um die Bindung zur meso-Position minimalisiert. Auch wenn hierbei wieder das Problem der Ungewißheit über die exakten Bindungslängen und Winkel auftritt, sowie die Tatsache, daß diese nicht energetisch günstig während der Rotation um $\Phi_{1}$ und $\Phi_{2}$ geändert werden konnten und den Bereich der energetisch möglichen Konformationen stark einengen, läßt der Verlauf der isoener- getischen Kurven eine deutliche Übereinstimmung mit den oben gemachten Konformationsbetrachtungen erkennen. Die drei lokalen Energieminima (und ihre entsprechenden spiegelbildlichen Anordnungen) des in der Abb. 22 dargestellten Ramachandran-Plots entsprechen den oben diskutierten Konformationen. Allerdings ist erstaunlich, da $\beta$ die relativen Energies der symmetrischen Konformation $\left(\Phi_{1}=\Phi_{2} \approx 20^{\circ}\right)$ so erheblich über dem absoluten Minimum liegen. Dies ist sicherlich zu einem groBen Teil auf die zur Berechnung verwendeten Bindungsverhältnisse zurückzuführen, wie schon oben diskutiert. Aber auch wenn diese verbessert werden, bleibt das globale Minimum der unsymmetrischen Konformation deutlich bevorzugt. Diese Konformation unterscheidet sich nur geringfügig von der aus dem Kalottenmodell abgeleiteten.

Die gleiche Rechnung zeigte für das methylsubstituierte Dimere 3d eine erhebliche sterische Behinderung, aus der eine starke Einschränkung der möglichen Konformationen resultiert. Aber auch hierfür ergab sich eine gute Übereinstimmung zwischen der aus dem Kalottenmodell abgeleiteten sterisch günstigsten und der berechneten energetisch

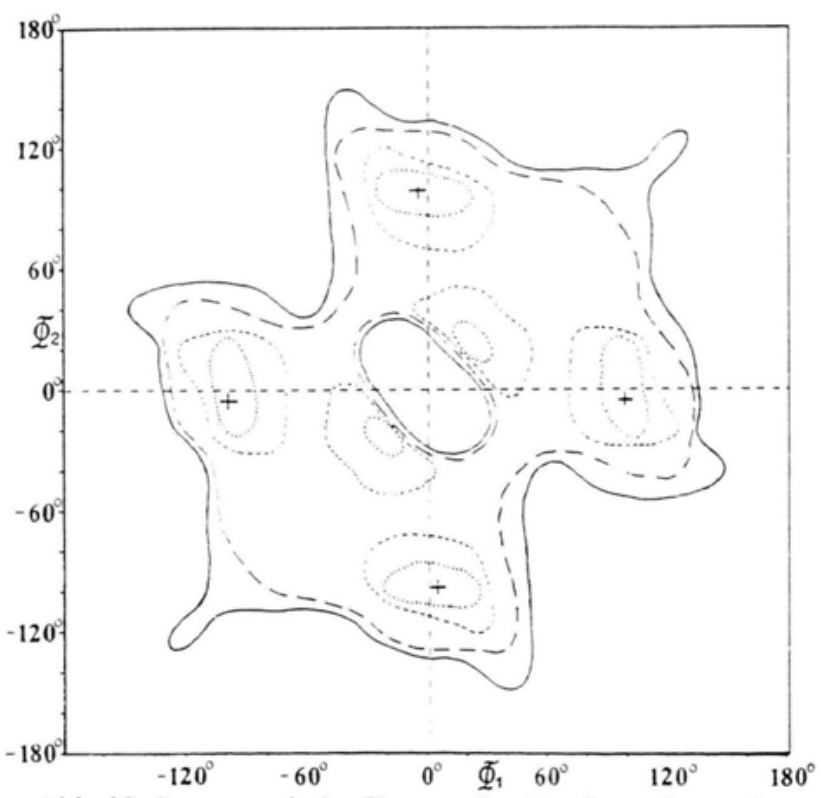

Abb. 22. Isoenergetische Kurven der Konformationen des Diporphinylmethans 3 a als Funktion der Torsionswinkel $\Phi_{1}$ und $\Phi_{2}$ in $20^{\circ}$-Schritten. Die markierten Positionen zeigen die energetisch günstigsten Konformationen an

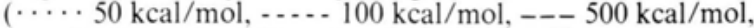
$1000 \mathrm{kcal} / \mathrm{mol}$ über dem Minimum (+)). 
günstigsten Konformation. Für diese wurde bei Energieminimalisierung der an der Methylenbrücke und der direkt dazu benachbarten meso-ständigen Methylgruppen $\Phi_{1}=99^{\circ}$ und $\Phi_{2}=-23^{\circ}$ errechnet, wie sie etwa auch in Abb. 14 a schematisiert dargestellt ist. $\left(\Phi_{1}\right.$ ist definiert über die Positionen $2^{\prime}$-Methylenbrücke-2-3 und $\Phi_{2}$ über $3^{\prime}$-2'-Methylenbrükke-2.)

Um dieses einfach zu handhabende Modell der Wechselwirkung ausgedehnter Dipole für dimere Porphine weiter zu überprüfen, wurden zwei weitere von Paine, Dolphin und Gouterman beschriebene [30] signifikant unterschiedlich $\beta, \beta^{\prime}$-verknüpfte Dimere des Etioporphyrin I untersucht.

Wie den Abb. 23 und 24 zu entnehmen ist, zeigen die vierwertigen Kationen deutlich aufgespaltene Soret-Banden. Da die Q-Banden dieser keine Abweichung gegenüber denen des monomeren Dikations der Ausgangsverbindung Etioporphyrin zeigten, folgt daraus, daß die Symmetrie des einzelnen Chromophors mit der des Monomer übereinstimmt. Nimmt man an, wie oben diskutiert, daß diese für OEP-, und andere nur $\beta$-alkylsubstituierte PorphinDikationen typische Q-Bandenform daher resultiert, daß diese Dikationen im Gegensatz zu denen der meso-substituierten Porphine planarer sind, kann dies auch für die hier diskutierten Dimeren des Etioporphyrins als Grundlage für die Konformationsbetrachtungen dienen.

Nach Kalottenmodellen ergibt sich für die günstigste Konformation des direkt $\beta, \beta^{\prime}$-verbundenen Dimeren eine senkrechte Anordnung der Porphinringe mit dem Torsionswinkel $\Phi=90^{\circ}$. Die daraus folgende Stellung der Dipole ergibt die in Abb. 23b wiedergegebene Superposition der dazugehörigen Teilbanden. Zum Vergleich wurden auch die abgebildet, die aus dem Torsionswinkel $\Phi=40^{\circ}$ bzw. $\Phi=130^{\circ}$ resultieren. Die für $\Phi=90^{\circ}$ berechnete Bandenform stimmt hervorragend mit der beobachteten überein und bestätigt die Anwendbarkeit dieses Modells.

Die Aufspaltung der Soret-Bande (Abb. 24a) des $\beta, \beta^{\prime}$-methylenverbrückten Dimeren gleicht der von $\mathbf{3 d}$ und $\mathbf{3} \mathbf{g}$; allerdings ist die Intensität der bathochromen Teilbande im Verhältnis zur hypsochromen deutlich geringer. Letztere weist auch eine intensive Schulter an der kurzwelligen Flanke auf. Die konformativen Möglichkeiten unterscheiden sich allerdings erheblich von denen der meso-methylsubstituierten Derivative 3 auf Grund der unsubsti- tuierten meso-Position sowie der zur Methylenbrücke benachbarten Methylgruppen in $\beta$-Position. Nach Kalottenmodellen ergeben sich zwei sterisch günstige Konformationen, $\Phi_{1}=\Phi_{2}= \pm 130^{\circ}$ bzw. $\Phi_{1}=60^{\circ}, \Phi_{2}=-125^{\circ}$. Aus erster resultiert ein Kurvenverlauf, der mit der beobachteten SoretBande besonders in Bezug auf die Intensitätsverhältnisse gut übereinstimmt (vgl. Abbildung $24 \mathrm{~b}$ ). Eine bessere Übereinstimmung ergibt sich, wenn man die Kopplung mit dem Faktor $k=1,6$ und nicht wie sonst mit $k=1,75$ berechnet. Mit der Konformation $\Phi_{1}=50^{\circ}, \Phi_{2}=-130^{\circ}$ und der sehr ähnlichen $\Phi_{1}=60^{\circ}, \Phi_{2}=-125^{\circ}$ erhält man ebenfalls eine Bandenform, die der beobachteten gleicht (Abbildungen 24c, d). Da in diesem Fall aus den sterisch günstigsten Konformationen sehr ähnliche Spektren berechnet werden, ist eine Entscheidung zu Gunsten einer schwierig. Trotzdem läßt sich aber auch hierfür eine befriedigende Übereinstimmung mit dem Modell feststellen.

An diesem Beispiel läßt sich auch der starke Einfluß der geringen Änderung der Halbwertsbreite der einzelnen Teilbanden auf die Form der durch die Superposition erzeugten Gesamtkurve zeigen: Die Abb. $24 \mathrm{~b}-\mathrm{d}$ zeigen die errechneten Bandenlagen und Intensitäten einmal mit der Grundkurve, die eine Halbwertsbreite von $\Delta \lambda_{1 / 2}=11 \mathrm{~nm}$ aufweist (wie beim Dikation des TMP 1a) und dann mit $\Delta \lambda_{1 / 2}=14 \mathrm{~nm}$, wie sie beim Etioporphyrindikation beobachtet wird [30].

Zusammenfassend läßt sich sagen, daß das halbquantitativ angewandte Modell der Wechselwirkung ausgedehnter Dipole bei den hier untersuchten vier, signifikant unterschiedlichen dimeren Porphinen eine gute Übereinstimmung von berechneter und beobachteter Aufspaltung der Soret-Bande der vierwertigen Kationen liefert. Die Form der aufgespaltenen Bande wird durch die energetisch günstigste Konformation der excitonengekoppelten Dimere bestimmt. Es ist nicht nur möglich eine Aussage über den durchschnittlichen Abstand der Porphine zu erhalten, wie etwa durch ESR-Messungen, sondern auch über die Stellung der Porphinebenen zueinander. Die Anwendung dieses Modells wird durch die einfache Handhabung gerechtfertigt.

Mein besonderer Dank gilt Herrn Professor Dr. H. Kuhn und Herrn Professor Dr. H. D. Försterling für die hilfreiche Diskussion bei der Anwendung dieses Modells. Herrn Dr. G. Barnickel sei besonders für die Zurverfügungstellung des Konforma- 

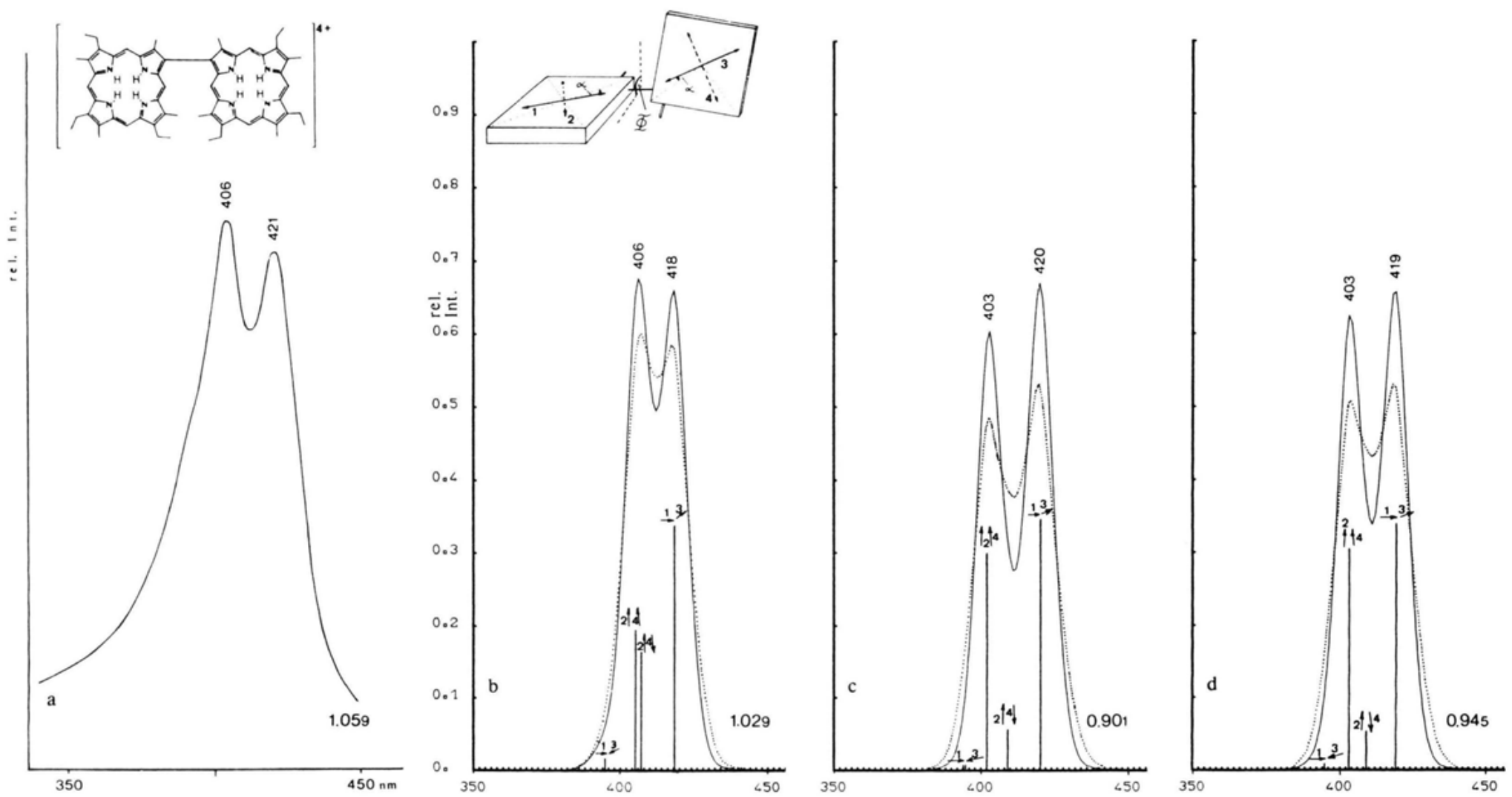

Abb. 23. Experimentelle a: in $\mathrm{CH}_{2} \mathrm{Cl}_{2} / \mathrm{CF}_{3} \mathrm{COOH}[30]$ und berechnete Soret-Banden des vierwertigen Kations des direkt in den $\beta$, $\beta^{\prime}$-Positionen verbundenen

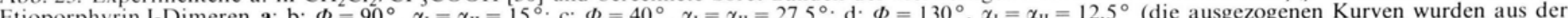
Superposition von Teilbanden mit einer Halbwertsbreite von $\Delta \lambda_{1 / 2} \approx 11 \mathrm{~nm}$ erhalten, die punktierten mit einer Halbwertsbreite von $\Delta \lambda_{1 / 2} \approx 14 \mathrm{~nm} ; k=1,75$ ). 

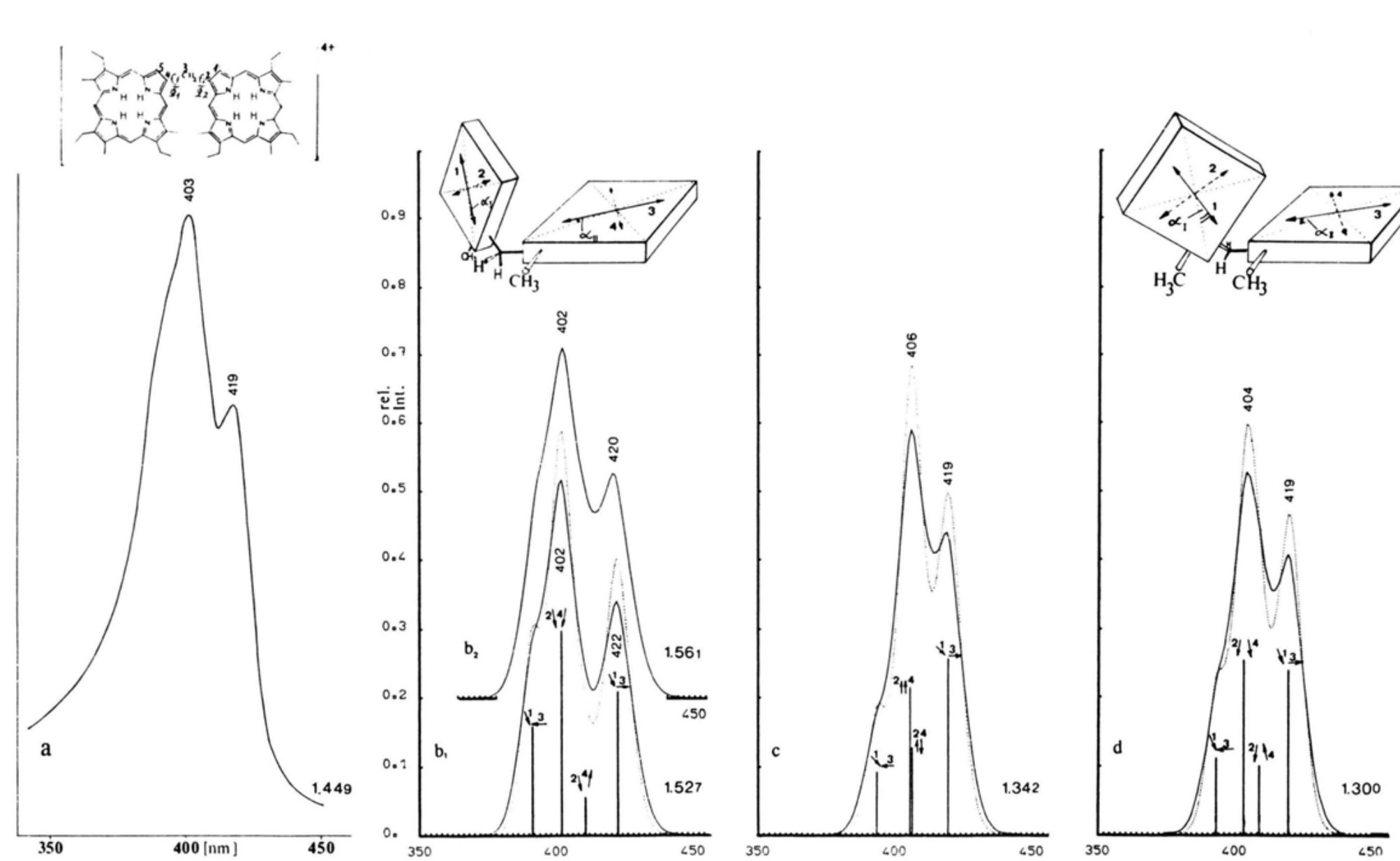

Abb. 24. Experimentelle a: in $\mathrm{CH}_{2} \mathrm{Cl}_{2} / \mathrm{CF}_{3} \mathrm{COOH}$ [30] und berechnete Soret-Banden des vierwertigen Kations des $\beta, \beta^{\prime}$-methylenverbrückten Etioporphyrin IDimeren b; b: $\Phi_{1}=\Phi_{2}=130^{\circ}, \alpha_{1}=\alpha_{11}=20^{\circ}$; c: $\Phi_{1}=50^{\circ}, \Phi_{2}=-130^{\circ}, \alpha_{1}=27^{\circ}, \alpha_{\| l}=10^{\circ}$; d: $\Phi_{1}=60^{\circ}, \Phi_{2}=-125^{\circ}, \alpha_{1}=25^{\circ}, \alpha_{I I}=15^{\circ}$. Während in $\mathrm{b}_{1}$ die Kopplung mit $k=1,75$ berechnet wurde, wurde in $\mathrm{b}_{2}$ der Wert $k=1,6$ angenommen. Der punktierte Kurvenverlauf ist die Superposition von Teilbanden mit einer Halbwertsbreite von $\Delta \lambda_{1 / 2} \approx 11 \mathrm{~nm}$, die durchgezogene mit $\Delta \lambda_{1 / 2} \approx 14 \mathrm{~nm} ; \lambda_{\text {mono }}=406 \mathrm{~nm} ; k=1,75$. (Der Torsionswinkel $\Phi_{1}$ ist definiert über die Positionen 2-3-4-5 und $\Phi_{2}$ über 1-2-3-4.) 
tions-Energie-Rechenprogrammes sowie seiner Hilfe gedankt.

\section{Experimenteller Teil und Anhang}

Die Elektronenspektren wurden mit dem Beckman-Gerät ACTA-MVI gemessen. Zur Vermessung der Neutralbasen wurde das $\mathrm{CHCl}_{3}$ (Uvasol) mit $\mathrm{Na}_{2} \mathrm{CO}_{3}$ (wasserfrei, p. a.) säurefrei gehalten.

Über die Synthesen und Analysen der Porphinderivate wurde an anderer Stelle berichtet [23, 33]. Zur Demetallierung der Nickelkomplexe wurden diese in $\mathrm{CHCl}_{3}$ gelöst. Die rotviolette Lösung wurde zu eisgekühlter $45 \% \mathrm{H}_{2} \mathrm{SO}_{4}$ gegeben, intensiv geschüttelt bis die Chloroformphase farblos und die Schwefelsäurephase intensiv türkisgrün war. Dann wurden beide Phasen gemeinsam vorsichtig bei $-15^{\circ} \mathrm{C}$ zu einer konzentrierten wäßrigen Ammoniaklösung (ca. $25 \%$, p. a.) gegeben. Diese bis zur Farblosigkeit mit $\mathrm{CHCl}_{3}$ ausgeschüttelt und die vereinigten Cloroformphasen über $\mathrm{Na}_{2} \mathrm{CO}_{3}$ eingeengt, anschließend diese Lösung dünnschichtchromatographisch (DCPlatten der Fa. Riedel-deHaen, Kieselgel SIF) mit $\mathrm{CHCl}_{3}\left(\right.$ ü. $\mathrm{Na}_{2} \mathrm{CO}_{3}$ ) getrennt.

Die erste Fraktion enthielt wenig des ursprünglichen Nickelkomplexes, die zweite Fraktion im Falle der Porphinderivate $\mathbf{1} \mathbf{b}, \mathbf{1} \mathbf{e}$, und $\mathbf{1} \mathbf{g}$ die Neutralbase. Bei den Dimeren $\mathbf{2} \mathbf{b}, \mathbf{3} \mathbf{b}, \mathbf{3} \mathbf{e}$ und $\mathbf{3 h}$ bestand die zweite Fraktion aus den entsprechenden monometallierten Komplexen und die violette dritte Fraktion aus den vollständig demetallierten Neutralbasen. Die $\mathrm{R}_{\mathrm{F}}-$ Werte $\left(\mathrm{CHCl}_{3}\right)$ betrugen für $\mathbf{1} \mathbf{a}$ $0,42, \mathbf{1}$ d $0,41, \mathbf{1}$ f $0,40,2$ a $0,44,3$ a $0,46,3$ c $0,61,3$ d $0,45, \mathbf{3} \mathbf{f} 0,62$ und für $\mathbf{3} \mathbf{g} 0,46$.

\section{Bestimmung der Bandenverschiebungen}

Die Berechnung der Bandenverschiebungen, die bei der Wechselwirkung zweier Porphinaromaten zu erwarten sind, erfolgte analog dem in der Literatur beschriebenen Weg [31]. Danach ist das Wechselwirkungsintegral $J_{1,2}$ bzw. die Verschiebung der Banden nur noch eine Funktion der Abstände $a_{1}$, $a_{2}, a_{3}$ und $a_{4}$ der beiden Dipole mit der Länge $l$ und der Ladung $\varepsilon$, wenn der Wert für $\varepsilon^{2} / D$ bekannt ist. $\mathrm{Zu}$ dessen Bestimmung wurden die experimentell ermittelten Daten eines Porphincyclophans mit kurzen $-\left(\mathrm{CH}_{2}\right)_{4}$-Brückengliedern verwendet [11], um eine möglichst parallele, nicht gewinkelte und zu- einander nicht verschobene Anordnung beider Porphinebenen zu gewährleisten. Es gilt (2),

$$
J_{1,2}=\frac{\varepsilon^{2}}{D}\left(\frac{1}{a_{1}}+\frac{1}{a_{2}}-\frac{1}{a_{3}}-\frac{1}{a_{4}}\right),
$$

weiterhin steht die Länge $/$ des ausgedehnten Dipols und die Ladung $\varepsilon$ mit dem Übergangsmoment $M$ über

$$
M=\varepsilon \cdot l
$$

in Beziehung. Ist $M$ und $J_{1,2}$ bekannt, so läßt sich $\varepsilon$ und $l$ aus (1) und (2) bestimmen, wenn man eine bestimmte Anordnung der beiden wechselwirkenden Moleküle vorgibt.

Für die planparallele Anordnung im Porphincyclophan ergibt sich:

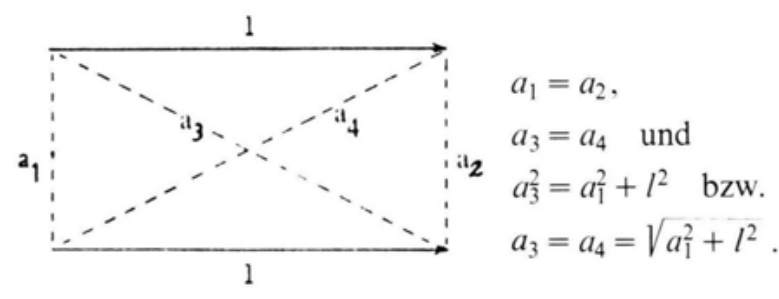

Hiermit errechnet sich nach (2) und (3)

$$
J_{1,2}=\frac{M^{2}}{l^{2} D} \cdot\left(\left(\frac{2}{a_{1}}\right)-\left(\frac{2}{\sqrt{a_{1}^{2}+l^{2}}}\right)\right) .
$$

\section{Bestimmung des Übergangsmomentes $M$ für das Dikation des TMP $\mathbf{1} \boldsymbol{a}$ und Ni-TMP $\mathbf{1} \boldsymbol{b}$ :}

Das Übergangsmoment $M$ der Soret-Bande läßt sich aus dessen Intensität $\varepsilon_{\max }$, der Halbwertsbreite $\Delta i_{1 / 2}$ sowie der Multiplizität $G=2$ nach

$$
M^{2}=\frac{\varepsilon_{\max } e_{0}^{2}}{2513 G} \frac{\Delta i_{1 / 2}}{\lambda}
$$

bestimmen; $e_{0}$ ist die Elementarladung.

Nach Tab. 1 ergibt sich für das Dikation von TMP 1 a in $\mathrm{CHCl}_{3} / 1 \% \mathrm{CF}_{3} \mathrm{COOH}$ mit $\varepsilon_{421}=343630$ und $\Delta \lambda_{1 / 2}=11,5 \mathrm{~nm} M=6,57$ Debye, in $\mathrm{H}_{2} \mathrm{SO}_{4}$ mit $\varepsilon_{413}=366500$ und $\Delta i_{1 / 2}=11 \mathrm{~nm} M=6,70$ Debye. Für Ni-TMP $\mathbf{1 b}$ errechnet sich mit $\varepsilon_{418}=185500$ und $\Delta \lambda_{1 / 2}=19 \mathrm{~nm} \quad M=6,22$ Debye. Vereinfachend bestimmt sich damit für $\mathrm{TMPH}_{4}^{2+}$ und Ni-TMP 1 b gemeinsam das Übergangsmoment zu

$$
M=6,5 \text { Debye } \text {. }
$$


Bestimmung von $J_{1,2}$

Aus (1) ergibt sich

$$
J_{1,2}=\frac{1}{2} h c\left(\frac{1}{i_{\text {dimer }}}-\frac{1}{i_{\text {mono }}}\right)
$$

$\left(h=6,625 \cdot 10^{-27} \mathrm{erg} \cdot \mathrm{sec}, c=2,997 \cdot 10^{10} \mathrm{~cm} \cdot \mathrm{sec}^{-1}\right)$.

Für das cyclophanartige vierwertige Kation des Dimeren wird $\lambda_{\text {dimer }}=412 \mathrm{~nm}$ beobachtet [11], für das entsprechende monomere Dikation kann man aus den experimentellen Daten $\lambda_{\text {mono }}=428 \mathrm{~nm}$ annehmen [11]. So errechnet sich

$$
J_{1,2}=0,09 \cdot 10^{-12} \mathrm{erg} \text {. }
$$

\section{Bestimmung der Länge l und der Ladung $\varepsilon$ des ausgedehnten Dipols}

Nach (4) ergibt sich mit dem aus einem Kalottenmodell für das vierwertige Kation abgeleiteten Ebenenabstand von $a_{1}=a_{2}=5,0 \AA$ und den Werten $M=6,5 \cdot 10^{-18} \mathrm{~g}^{1 / 2} \cdot \mathrm{cm}^{5 / 2} \cdot \mathrm{s}^{-1}, J_{1,2}=0,09 \cdot 10^{-12} \mathrm{~cm}^{2}$ $\cdot \mathrm{g} \cdot \mathrm{s}^{-2}$ und $D=2,5$ für die Länge $l$ des ausgedehnten Dipols

$$
l=4,05 \AA
$$

und aus (3) folgt für die Ladung $\varepsilon$ des ausgedehnten Dipols

$$
\varepsilon=0,331 e_{0} .
$$

Zwei Beispiele zur Fehlerbetrachtung der Bestimmung der Länge l und der Ladung \& des ausgedehnten Dipols

a) mit $M=(6,44+5 \%)$ Debye, $a_{1}=(5-2 \%) \AA$, $D=(2,5-10 \%)$ und unverändertem $J_{1,2}=0,09 \cdot 10^{-12}$ erg ergibt (4) $l=5,6 \AA$, und daraus folgt $\varepsilon=0,25 e_{0}$.

b) Mit $M=(6,44-5 \%)$ Debye, $a_{1}=(5+2 \%) \AA$, $D=(2,5+10 \%)$ und $J_{1,2}=0,09 \cdot 10^{-12}$ erg errechnet sich nach (4) $l=2,2 \AA$ und $\varepsilon=0,58 e_{0}$.

Mit (2) ergibt (1) bei Einführung des Korrekturfaktors $k$

$$
\frac{1}{\lambda_{\text {dimer }}}=\frac{k\left( \pm 2 \frac{\varepsilon^{2}}{D}\right)}{h c}\left(\frac{1}{a_{1}}+\frac{1}{a_{2}}-\frac{1}{a_{3}}-\frac{1}{a_{4}}\right)+\frac{1}{\lambda_{\text {mono }}},
$$

womit die hier diskutierten Aufspaltungen berechnet wurden.

Rechenbeispiel für das dimere vierwertige Kation der Struktur 3 mit der Konformation, die in Abb. 14d dargestellt ist

Definition von $\Phi_{1}$ und $\Phi_{2}$ :

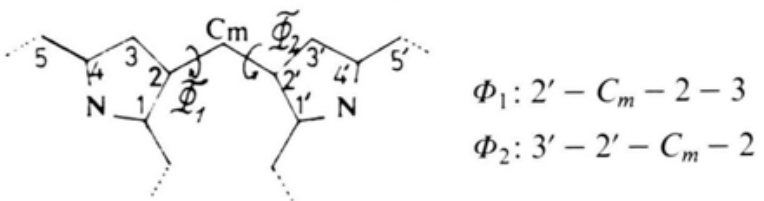

$\Phi_{1}=70^{\circ} ; \Phi_{2}=-30^{\circ} ; l=4,05 \AA ; \varepsilon=0,331 e_{0}, D=$ 2,5 (Mittelpunktsabstand der beiden Porphinringe $9,76 \AA$ ). Wenn $\alpha_{I}=7^{\circ}$ und $\alpha_{I I}=10^{\circ}$, besteht keine Kopplung zwischen den Dipolpaaren 1-4 und 2-3. Für die Abstände erhält man dann aus dem Modell für das Dipolpaar 1-3: $a_{1}=9,94 \AA, a_{2}=9,96 \AA$, $a_{3}=6,3 \AA, a_{4}=13,24 \AA$; daraus errechnet sich nach (6) mit $k=1,75$ und $\lambda_{\text {mono }}=428 \mathrm{~nm}$ für die, ,inPhase"-Anordnung $i \overrightarrow{\text { dimer }}=439,1 \mathrm{~nm}$ mit der relativen Intensität $f=30,6$ und für die ,gegen-Phase". Anordnung $i \underset{\text { dimer }}{\rightleftarrows}=417,4 \mathrm{~nm}$ mit $f=10,4$. Für das Dipolpaar $2-4$ erhält man $a_{1}=9,88 \AA, a_{2}=9,9 \AA$, $a_{3}=11,0 \AA, a_{4}=10,0 \AA$ und damit für die ,inPhase"-Anordnung $/ \overrightarrow{\text { dimer }}=424,3 \mathrm{~nm}$ mit $f=37,25$ und für die ,gegen-Phase“-Anordnung i i $\mathrm{nm}$ mit $f=3,7$ (vgl. Abbildung 14d).

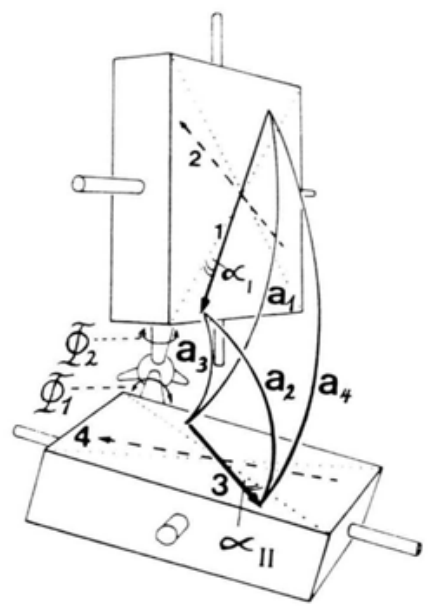


[1] G. Renger in Biophysik, Ein Lehrbuch (W. Hoppe, W. Lohmann, H. Markl, H. Ziegler), 1. Aufl., S. 415, Springer-Verlag, Berlin 1978.

[2] J. M. Olson in The Photosynthetic Bacteria (R. K. Clayton, W. R. Sistrom), S. 161, Plenum Press, New York 1978

[3] T. Miyasaka, T. Watanabe, A. Fujishima u. K. Honda, J. Amer. Chem. Soc. 100, 6657 (1978).

[4] A. F. Janzen, J. R. Bolton u. M. J. Stillman, J. Amer. Chem. Soc. 101, 6337 (1979).

[5] G. M. Maggiora, Int. J. Quantum Chem. 16, 331 (1979).

[6] K. Colbow u. R. P. Dunyluk, Int. J. Quantum Chem., Quantum Biology Symp. No. 3, 151 (1976).

[7] T. L. Netzel, M. A. Bergkamp u. C. K. Chang, J. Amer. Chem. Soc. 1982, 104 (1952).

[8] A. Warshel, J. Amer. Chem. Soc. 101, 744 (1979).

[9] H. Scheer u. J. J. Katz, Porphyrins and Metalloporphyrins (K. M. Smith), S. 399, Elsevier, Amsterdam 1975.

[10] J. P. Collman, P. Denisevich, Y. Konai, M. Marrocco, C. Koval u. F. C. Anson, J. Amer. Chem. Soc. 1980, $102,6027-6036$.

[11] J. P. Collman, A. O. Chong, G. B. Jameson, R. T. Oakley, E. Rose, E. R. Schmittou u. J. A. Ibers, J. Amer. Chem. Soc. 1981, 103, 516-533.

[12] C. K. Chang, J. Heterocyclic Chem. 14, 1285 (1977).

[13] J. P. Collman, C. M. Elliot, T. R. Halbert u. B. Tovrog, Proc. Natl. Acad. Sci. USA 74, 18 (1977).

[14] C. K. Chang, M.-S. Kuo u. C.-B. Wang, J. Heterocyclic Chem. 14, 943 (1977).

[15] N. E. Kagan, D. Mauzerall u. R. B. Merrifield, J. Amer. Chem. Soc. 99, 5484 (1977).

[16] M. R. Wasielewski, W. A. Svec u. B. T. Cope, J. Amer. Chem. Soc. 100, 1961 (1978).

[17] C. K. Chang, J. C. S. Chem. Comm. 1977, 800.

[18] H. Ogoshi, H. Sugimoto u. Z. Yoshida, Tetrahedron Lett. 1977, 169 .

[19] D. P. Arnold, A. W. Johnson u. M. Mahendran, J.C.S. Perkin I 1978, 366.

[20] D. Arnold, A. W. Johnson u. M. Winter, J.C.S. Perkin I 1977, 1643.

[21] R. G. Little, J. Heterocyclic Chem. 15, 203 (1978).

[22] J. B. Paine III u. D. Dolphin, Can. J. Chem. 56, 1710 (1978).

[23] B. v. Maltzan, Liebigs Ann. Chem. 1978, 238.

[24] St. G. Boxer u. R. R. Bucks, J. Amer. Chem. Soc. 101, 1883 (1979).

[25] J. A. Anton, J. Kwong u. P. A. Loach, J. Heterocycl. Chem. 13, 717 (1976).

[26] K. Ichimura, Chem. Lett. Japan 1977, 641.

[27] G. Meyer u. D. Wöhrle, Z. Naturforsch. 32 b, 723 (1977).

[28] M. Gouterman, D. Holten u. E. Liebermann, Chem. Phys. 25, 139 (1977).

[29] G. Meyer, M. Hartmann u. D. Wöhrle, Makromol. Chem. 176, 1919 (1975).
[30] J. B. Paine III, D. Dolphin u. M. Gouterman, Can. J. Chem. 56, 1712 (1978).

[31] V. Czikkeley, H. D. Försterling u. H. Kuhn, Chem. Phys. Letters 6, 207 (1970).

[32] B. v. Maltzan, unveröffentlicht.

[33] B. v. Maltzan, Liebigs Ann. Chem. 1980, 1082.

[34] N. Datta-Gupta u. T. J. Bardos, J. Heterocyclic Chem. 3,495 (1966)

[35] M. Gouterman in The Porphyrins (D. Dolphin), Bd. III, Teil A, 1. Aufl., S. 13, Academic Press, New York 1978.

[36] J. V. Knop u. A. Knop, Z. Naturforsch. 25 a, 1720 (1970).

[37] K. N. Solovev, Optics Spectroscopy 10, 389 (1961); A. N. Sevchenko, K. N. Solovev, V. A. Mashenkov u. S. F. Shkirman, Soviet Phys.-Dokl. Acad. Sci. 10, 778 (1966).

[38] G. M. Maggiora, J. Amer. Chem. Soc. 95, 6555 (1973).

[39] J. D. Pethke, G. M. Maggiora, L. L. Shipman u. R. E. Christoffersen, J. Mol. Spectr. 71, 64 (1978).

[40] M. Kasha, H. R. Rawls u. M. Ashraf El-Bayoumi, Pure Appl. Chem. 11, 371 (1965).

[41] H. D. Försterling u. H. Kuhn, persönliche Mitteilung.

[42] G. Barnickel, Fortran-Program for the Calculation of the Conformational-Potential Energy (CPE), Institut für Kristallographie der Freien Universität Berlin, 1982.

[43] A. Ulman, J. Galluci, D. Fisher u. J. A. Ibers, J. Amer. Chem. Soc. 1980, 102, 6852-6854.

[44] D. L. Cullen u. E. F. Meyer, J. Amer. Chem. Soc. 96, 2095 (1974).

[45] J. Moustakli u. A. Tulinsky, J. Amer. Chem. Soc. 95, 6811 (1973).

[46] G. Kortüm, Lehrbuch der Elektrochemie, 5. Aufl., S. 208, Verlag Chemie, Weinheim 1970.

[47] H. D. Försterling u. H. Kuhn, Int. J. Quant. Chem. 2, 413 (1968).

[48] H. Kuhn, Chimia 15, 53 (1961).

[49] B. Evans, K. M. Smith u. J.-H. Fuhrhop, Tetrahedron Lett. 1977, 443

[50] A. Stone u. E. B. Fleischer, J. Amer. Chem. Soc. 90, 2735 (1968)

[51] E. Cetinkaya, A. W. Johnson, M. F. Lappert, G. M. McLaughlin u. K. W. Muir, J. Chem. Soc. Dalton $1236,1974$.

[52] P. W. Codding u. A. Tulinsky, J. Amer. Chem. Soc. 94, 4151 (1972).

[53] T. R. Janson u. J. J. Katz in The Porphyrins (D. Dolphin), Band IV, S. 25, Academic Press, London 1978.

[54] B. v. Maltzan, Angew. Chem. 94, 801 (1982).

[55] Lit. [46], S. 171.

[56] Lit. [46], S. 219.

[57] L. P. Hammett, Physikalische Organische Chemie, S. 303, Verlag Chemie, Weinheim 1973. 